\title{
Le réseau Salomé (Vallon-Pont-d'Arc, Ardèche, France) : un nouvel exemple de compétition et d'interactions interspécifiques (ours-hyène)
}

Réseau Salomé (Vallon-Pont-d'Arc, Ardèche, France): a new case of interspecific interactions between bears and hyenas

Jean-Baptiste Fourvel, Michel Philippe, Jacqueline Argant et Nicolas Lateur

\section{OpenEdition}

Journals

Édition électronique

URL : http://journals.openedition.org/paleo/3539

DOI : $10.4000 /$ paleo.3539

ISSN : $2101-0420$

Éditeur

SAMRA

Édition imprimée

Date de publication : 30 décembre 2017

Pagination : 227-249

ISSN : $1145-3370$

Référence électronique

Jean-Baptiste Fourvel, Michel Philippe, Jacqueline Argant et Nicolas Lateur, « Le réseau Salomé (Vallon-Pont-d'Arc, Ardèche, France) : un nouvel exemple de compétition et d'interactions

interspécifiques (ours-hyène) ", PALEO [En ligne], 28 | 2017, mis en ligne le 01 juin 2018, consulté le 07 juillet 2020. URL : http://journals.openedition.org/paleo/3539 ; DOI : https://doi.org/10.4000/paleo. 3539

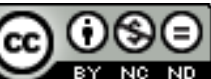

PALEO est mis à disposition selon les termes de la licence Creative Commons Attribution - Pas d'Utilisation Commerciale - Pas de Modification 4.0 International. 


\title{
Le réseau Salomé (Vallon-Pont-d'Arc, Ardèche, France) : un nouvel exemple de compétition et d'interactions interspécifiques (ours-hyène)
}

\author{
Jean-Baptiste FOURVEL(a), Michel PHILIPPE(b), \\ Jacqueline ARGANT ${ }^{(\mathrm{c})}$, Nicolas LATEUR ${ }^{(\mathrm{d})}$
}

\begin{abstract}
Résumé : Les interactions entre les différentes communautés animales, coexistant au sein des mêmes environnements, sont bien connues et ce tout au long du Quaternaire. Qu'il s'agisse d'interactions de type prédateur-proie (prédation, charognage) ou prédateur-prédateur (compétition), les ensembles archéologiques et paléontologiques renferment de nombreux indices de ces relations (taphonomie sensu lato). Partageant un même fonds écologique (proie, habitat) les guildes de prédateurs entrent régulièrement en compétition. Ces phénomènes se traduisent au sein des ensembles fossiles par toute une diversité d'éléments : des modifications de surface des os (traces de dents), des morphotypes de fracturations ou encore des bioglyphes (coprolithes). Au sein des guildes de Carnivores pléistocènes, le cas des Ursidés est questionné : s'agit-il de grands prédateurs entrant en compétition avec les autres espèces prédatrices ou sommesnous face à une espèce-proie régulièrement chassée et consommée par les autres carnivores (lion, hyène principalement) ? Le réseau Salomé (Vallon-Pont-d'Arc, Ardèche) est un nouvel exemple de ces relations et interactions entre les différentes communautés de grands carnivores (ours et hyène). À partir de l'examen paléontologique et taphonomique, ce site d'hivernation à ours des cavernes nous apporte de nouveaux éléments de discussion en vue de préciser le statut paléoécologique de l'ours des cavernes et sa position au sein des réseaux trophiques pléistocènes.
\end{abstract}

Mots-clés : Réseau Salomé, Ardèche, Pléistocène supérieur, Paléoécologie, Carnivores, hyène des cavernes, ours des cavernes.

\begin{abstract}
Réseau Salomé (Vallon-Pont-d'Arc, Ardèche, France): a new case of interspecific interactions between bears and hyenas. Inter-specific interactions in mammalian communities, which coexist within the same ecosystems, are well-know all along the Quaternary. Evidences of these relations between fauna (taphonomy sensu lato), including both predator-prey (e.g., hunting, scavenging) and predator-predator (e.g., competition) interactions, are regularly found in archaeological and palaeontological assemblages. Evolving in the same ecosystem, predator guilds are frequently in competition for food and habitat access. Within fossil samples, these competition phenomena are revealed by diverse aspects : bone surface modifications (e.g., tooth marks), bone morphotypes and bioglyphes (e.g., coprolithes). Within the Pleistocene carnivore guilds, Ursids' status is questioned : are they large predator which compete for food and habitat access or prey-species frequently hunted or scavenged by other carnivore species (e.g., lion, hyena). Réseau Salomé (Vallon-Pont-d'Arc, Ardèche) is a new example of these relations and interactions between various carnivore communities (bears and hyenas). Based on palaeontological and taphonomical analyses, we discuss here the cave bear's palaeoecological status and its position within the pleistocene trophic chain.
\end{abstract}

Key-words: Réseau Salomé, Ardèche, Late Pleistocene, Palaeoecology, Carnivores, Cave hyena, Cave bear.

(a) UMR5608 TRACES - GDR3591 TaphEnA - Université de Toulouse-Jean Jaurès, 5 Allée Antonio-Machado, FR-31058 Toulouse cedex 9 - jbfourvel@yahoo.com.

(b) Conservateur honoraire du Muséum de Lyon (Musée des Confluences) - mipauphi@wanadoo.fr

(c) UMR7269 LAMPEA, Maison Méditerranéenne des Sciences de l'Homme, 5 Rue du Château de l'Horloge, BP 647, FR-13094

Aix-en-Provence cedex 2 - j.argant@wanadoo.fr

(d) UMR7269 LAMPEA, Maison Méditerranéenne des Sciences de l'Homme, 5 Rue du Château de l'Horloge, BP 647, FR-13094

Aix-en-Provence cedex 2 - nico.lateur@yahoo.fr 


\section{1 - Introduction}

Se déployant dans le quart sud-est de l'Ardèche et le nord du Gard, le plateau des Gras constitue une large entité calcaire, où le modelé karstique - aux diverses formes endo- et exokarstiques - a permis la conservation de nombreux sites archéologiques, témoignant de l'intérêt des groupes humains pour cette région située entre le sud-est du Massif central, la vallée du Rhône et le nord du Languedoc et de la Provence (Debard 1988). Au sein de cette entité, les gorges de l'Ardèche et les plateaux qui les bordent - celui de Saint-Remèze, au nord, et du Bois de Ronze, au sud - présentent une importante concentration de sites archéologiques et paléontologiques pléistocènes, dont les plus anciennes occupations humaines régionales sur le site d'Orgnac 3, datées du MIS 9 (Michel et al. 2013). La diversité de la topographie de ce secteur - les grands plateaux calcaires, les escarpements des gorges, la plaine alluviale rhodanienne à l'est, les nombreuses vallées incisant les contreforts cévenoles à l'ouest ou encore l'existence d'un réseau hydrographique pérenne - ont permis le développement des faunes pléistocènes, où la prégnance des grands Carnivores est remarquable, tant par le nombre d'occurrences que par la diversité des taxons de cette guilde (Debard et Philippe 2008 ; Lateur 2010 ; Philippe et Lateur 2013 ; Fourvel et al. 2016). On retrouve l'ensemble de ces grands Carnivores sous forme de restes osseux dans de nombreux sites archéologiques et/ou paléontologiques : à l'abri des Pêcheurs (Balme 1984), l'aven Flahaut (Debard et al., 1999), la grotte du Figuier (Moncel et al. 2012), l'aven de l'Arquet (Gambéri et al. 2011) et l'ensemble F de la grotte de Payre (Daujeard et Moncel, 2010), entre autres.

Parmi ceux-ci, les Ursidés et les Hyénidés sont particulièrement abondants. Les ours pléistocènes, en particulier, sont représentés par plusieurs taxons : l'ours du Tibet (Ursus tibethanus) à Orgnac 3 (Arouaghe 1992 ; Quilès 2003), l'ours de Deninger (Ursus deningeri) dans le même site ainsi qu'à la grotte des Fées (Ballesio et al. 2003), l'ours brun (Ursus arctos) dont un crâne a été observé à la grotte Chauvet (Philippe et Fosse 2003 ; Fosse et Philippe 2005), mais aussi présent à Orgnac 3, l'aven de l'Arquet, l'aven Flahaut ou encore la grotte du Figuier (Arouaghe 1992 ; Quilès 2003 ; Gambéri et al. 2011 ; Debard et al. 1999 ; Moncel et al. 2012). Mais c'est indubitablement l'ours des cavernes (Ursus spelaeus) qui a livré le plus d'occurrences. De très nombreuses cavités des gorges de l'Ardèche ont servi de gîtes pour l'hivernation (Philippe 2005), et dans lesquelles, en plus des restes paléontologiques, tous les bioglyphes témoignant de la fréquentation des réseaux profonds par ces Ursidés sont observables : bauges, griffades, polis, empreintes (parfois sous forme de pistes). Les Hyénidés forment eux-aussi une famille bien présente dans cette région, une synthèse récente ayant permis de souligner leur importance et de documenter les différents contextes dans lesquels ils sont identifiés (Fourvel et al. 2016). À noter que si l'hyène des cavernes (Crocuta crocuta spelaea) est l'espèce presque exclusive, la découverte récente d'une mandibule de Pachycrocuta brevirostris dans les dépôts de la grotte de la
Grosse-Marguerite (Aiguèze, Gard) atteste de la richesse des faunes pléistocènes locales (Fourvel et Lateur 2015).

Les Carnivores partageant avec les communautés humaines le même fonds écologique, parfois les mêmes habitats (grottes et abris-sous-roche) et les mêmes proies (Ongulés), les interactions directes entre Homme et Carnivores sont régulièrement mentionnées (Fourvel et al., 2016). Néanmoins, malgré les nombreux sites et contextes où se côtoient les différents Carnivores, leurs interactions sont, quant à elles, extrêmement mal documentées. En ce sens, l'éthologie de l'hyène des cavernes, notamment, dont on sait que son spectre alimentaire peut comprendre aussi bien des Ongulés que des Carnivores (synthèse in Fourvel 2012), est très mal connue.

La découverte en 2010 d'un nouveau gisement, le réseau Salomé (Vallon-Pont-d'Arc), où l'ours des cavernes et l'hyène des cavernes dominent largement l'assemblage et où l'impact taphonomique sur les restes d'ours est remarquable, a autorisé une analyse détaillée des relations et interactions prédateur-prédateur.

\section{2 - Le gisement, contexte et historique des recherches}

Peu avant sa confluence avec l'Ardèche à l'entrée des gorges, l'lbie est bordée, en rive gauche, de falaises escarpées dans lesquelles s'ouvrent de nombreuses grottes qui, pour la plupart, recèlent des gisements archéologiques et/ou paléontologiques. Trois d'entre elles (les grottes de Louoï, du Chasserou et du Déroc) sont des grottes ornées paléolithiques. Ces cavités se sont aussi révélées être d'importantes " grottes à ours » (Philippe 2005). C'est dans ce secteur que se situe le réseau Salomé, en liaison directe avec la grotte du Déroc et certainement aussi avec la grotte du Chasserou (même si la jonction n'a volontairement pas encore été effectuée) (fig. 1 et 2).

Malgré une importante fréquentation de la grotte du Déroc (spéléologues et visiteurs), le réseau Salomé n'a été repéré, par des membres du Spéléo-Club d'Aubenas, qu'en octobre 2010. Suite à la découverte d'abondants ossements, l'un de nous (MP) a été sollicité pour expertise et la mise en place d'une campagne de fouilles. Une unique campagne de terrain a été effectuée en août 2012, sous sa direction. En dépit de l'abondance du matériel paléontologique, aucun indice de fréquentation humaine n'a été observé. L'ensemble des vestiges non-enfouis observé et prélevé sur 12 loci, répartis sur une vingtaine de mètres carrés, a été repéré sur plan avant prélèvement. Par la suite, un sondage a été entrepris $\left(4 \mathrm{~m}^{2}\right)$ et étendu, permettant la mise au jour, l'enregistrement et la collecte de l'essentiel de la faune. Toutefois, après les premiers décapages, le sondage s'est concentré sur le carré B6, situé à proximité de la paroi nord de la Salle aux Os, celuici livrant une forte concentration osseuse (fig. 3). Le matériel a été coordonné dans les trois dimensions. Les sédiments très argileux n'ont pu être tamisés sur place. 


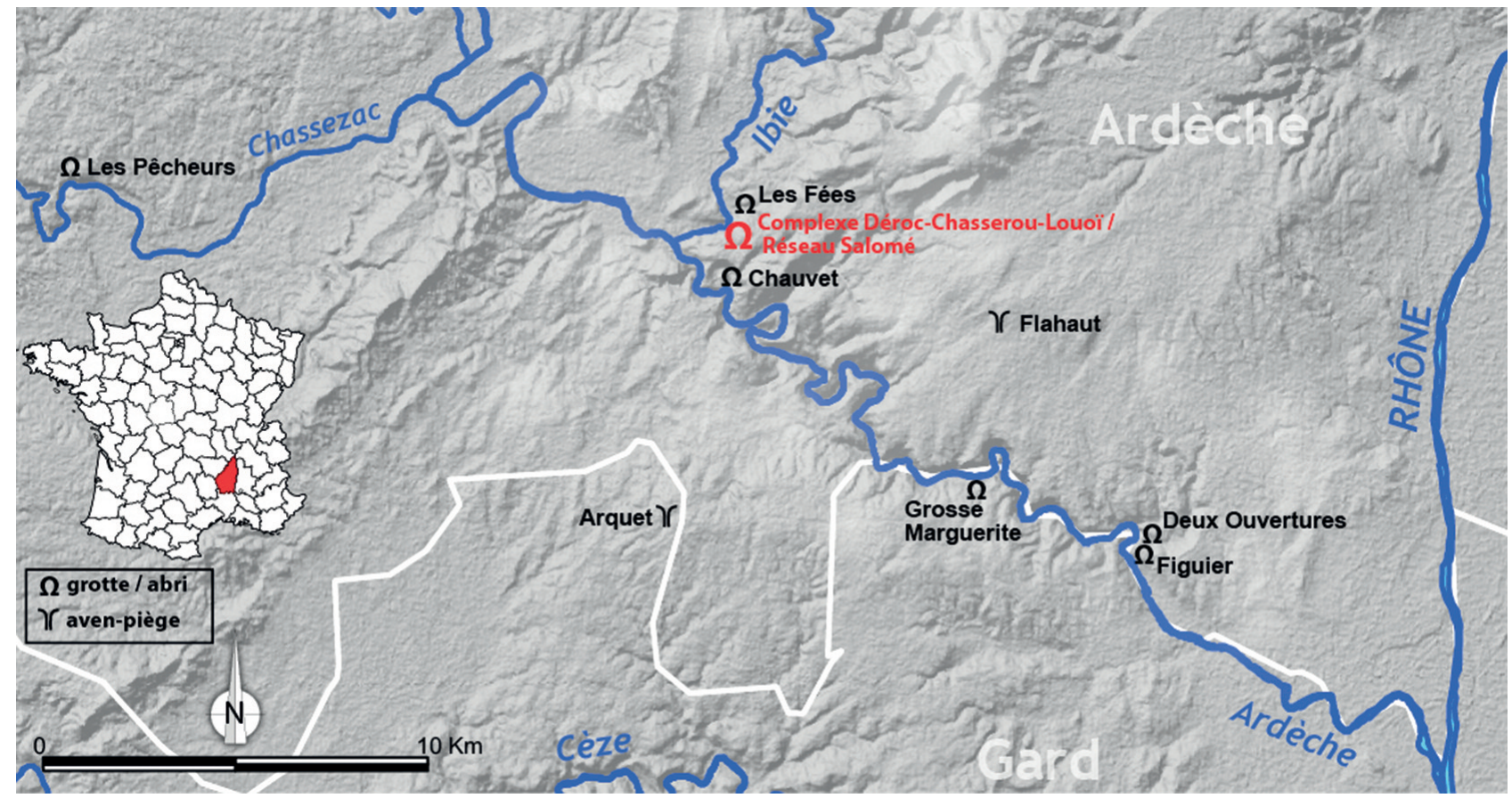

Figure 1 - Localisation du gisement du réseau Salomé. DAO N. Lateur.

Figure 1 - Geographical location of Réseau Salomé. Map realized by N. Lateur.

Cette opération a donc eu lieu a posteriori en laboratoire (tamisage à l'eau, maille $1,91 \mathrm{~mm}$, tri dans le tamis) et n'a fourni que quelques rares fragments osseux, en rien comparable avec les fortes concentrations d'esquilles observables dans certains repaires d'hyènes. Des coprolithes d'hyène des cavernes ont été découverts non seulement en plusieurs endroits du réseau mais aussi en stratigraphie dans le sondage, attestant a priori d'une accessibilité du réseau par les grands mammifères. Au total, ce sont 654 ossements, dents et coprolithes qui ont été prélevés dans le réseau Salomé, la plupart en surface de la Salle aux Os et en stratigraphie dans le sondage.

\section{3 - Matériel et méthode}

Si le réseau Salomé n'a, pour l'heure, pas encore fait l'objet d'études géomorphologiques permettant de préciser le contexte d'accumulation du gisement paléontologique, nous estimons toutefois que cela n'interdit pas l'étude proposée ici. Le matériel faunique observé en stratigraphie et en surface de la Salle aux Os, mais aussi dans les différents loci alentours, présente les mêmes caractéristiques en ce qui concerne les taxons et les éléments paléontologiques (sensu lato, i.e. dents, ossements et coprolithes) représentés. Par ailleurs, si les datations absolues n'ont pas offert de résultats précis, les datations réalisées sur des restes en surface dans un des loci et en stratigraphie dans la Salle aux os suggèrent un âge supérieur à 45000 ans. Enfin, les éléments de biochronologie disponibles (cf. infra) ne mettent pas non plus en évidence une diachronie préjudiciable à l'analyse et à son interprétation. Dans l'attente d'une analyse géomorphologique fine afin d'affiner notre analyse paléoécologique, nous considérons l'ensemble osseux comme un assemblage unique avec, cependant, des limites interprétatives.

L'étude que nous proposons se fonde sur l'examen paléontologique et taphonomique d'un échantillon ostéologique de 581 restes osseux rapportés à trois grands Carnivores (ours, lion, hyène) (tabl. 1). Une cinquantaine de pièces collectée dans 12 loci (nommés $A$ à $L$ ), adjacents à la zone de fouilles ou distants de celle-ci de plusieurs mètres, parfois sur l'étage de galeries supérieur, complète cet inventaire. D'autres taxons ont été déterminés par quelques restes. Un Capriné (bouquetin) est représenté par deux restes en stratigraphie (un cylindre de métacarpe et une incisive) et trois hors-stratigraphie (un talus droit, une première phalange et une portion de scapula). Notons la présence d'un sésamoïde de grand Bovidé et de deux dents jugales de Léporidé issus du sondage. Le renard roux Vulpes vulpes n'a été identifié que par une moitié proximale de radius au sein du Locus $\mathrm{F}$.

Afin de traiter ce matériel, trois unités de quantifications ont été utilisées : le Nombre de Restes (NR) total (NRT) ou déterminés (NRD), le Nombre Minimum d'Eléments (NME) et le Nombre Minimum d'Individus (NMI). Les résultats exprimés en NMI (et \%NMI) sont privilégiés ; le calcul d'un NMI limite les biais induits par un NR qui peut entraîner une surreprésentation déformant la réalité paléontologique. L'étude de cet assemblage porte sur deux aspects distincts : l'analyse paléontologique et l'analyse paléobiologique (intégrant l'examen taphonomique). 


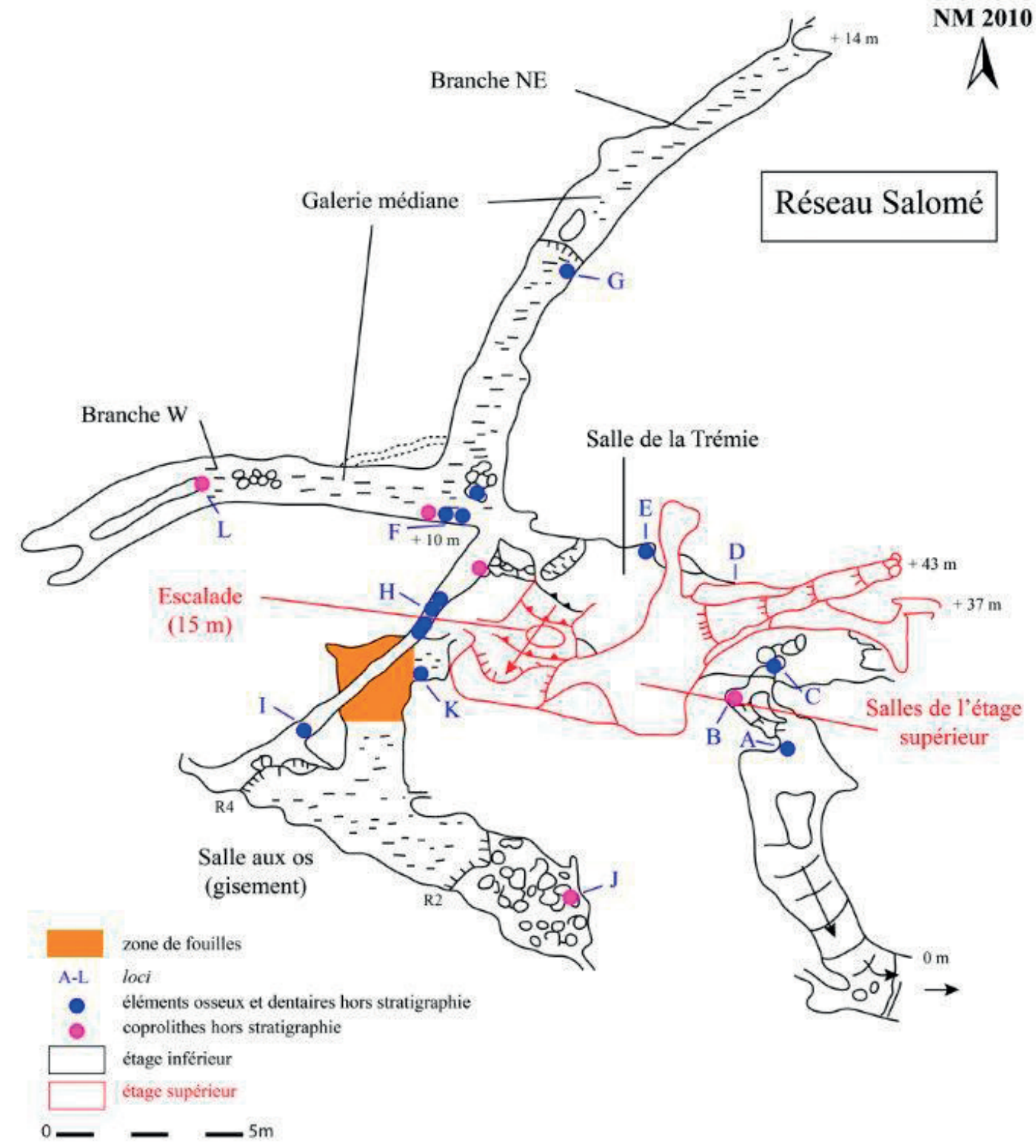

Figure 2 - Topographie du réseau Salomé. Localisation des loci et coprolithes. DAO N. Lateur et S. Stocchetti.

Figure 2 - Site topography. Location of bone samples and coprolithes. Digitalized map realized by N. Lateur and S. Stocchetti.

Différentes méthodes sont développées ou employées pour traiter chacun de ces aspects.

L'analyse paléontologique des grands Carnivores repose sur un examen morphométrique s'appuyant à la fois sur les protocoles de mesures spécifiquement développés pour l'étude de cet ordre (e.g., Clot 1980 ; Argant 1991) et sur l'analyse comparée d'autres populations issues de gisements a priori sub-contemporains ou plus anciens (e.g., Châtillon-Saint-Jean in Chauviré 1962 ; Mosbach in Kurtén 1962, 1972 ; Lunel-Viel 1 in Bonifay 1971, Cardoso 1993 ; Fouvent et Conives in Fourvel 2012).
L'analyse de la population d'hyène des cavernes a été réalisée à partir du matériel dentaire et du matériel postcrânien. La synthèse des différents travaux portant sur les séquences d'éruption et d'usure dentaire (détails in Fourvel 2012) nous amène à considérer cinq stades : 1) 0-1an : omniprésence des déciduales (éruption, usure puis chute des dents de lait) ; 2) 1-2,5 ans : sub-adulte avec denture définitive en éruption mais non usée ; 3) 4-6 ans : denture définitive en place et active (léger degré d'usure) ; 4) 6-10 ans : adulte âgé avec des couronnes largement usées ; 5) 10-15 ans/+ : sénile avec denture usée à son degré le plus fort (arasement complet des couronnes). 


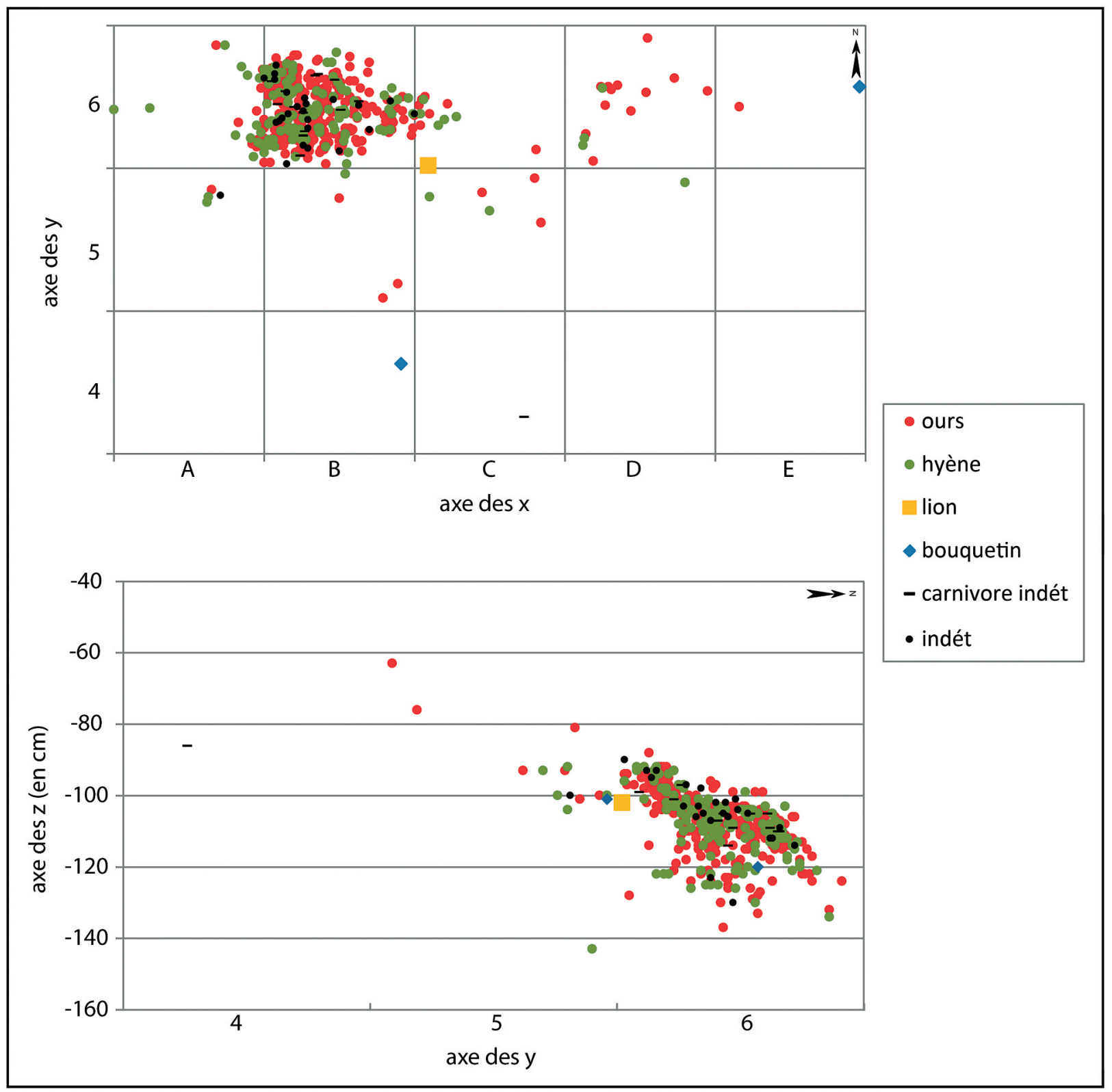

Figure 3 - Répartition des restes fauniques mis au jour dans le sondage de la Salle aux Os. DAO : N. Lateur

Figure 3 - Distribution of faunal remains from the excavation in the Salle aux Os. Digitalized by N. Lateur

En ce qui concerne le matériel postcrânien, en l'absence de travaux existant sur la croissance des éléments squelettiques et la synostose chez les Hyénidés, nous considérons quatre ensembles : 1) néonataux : os très poreux et de taille minimale ; 2) juvéniles : os en cours de croissance, moins poreux, mieux formé, absence des extrémités ; 3) sub-adultes : os au maximum de sa croissance (taille) et extrémités non soudées ou en cours de synostose ; 4) adultes et + : os totalement développé et synostose aboutie (aucune marque de fusion osseuse). Par ailleurs, nous avons appliqué ces quatre classes d'âge à la population ursine.
L'analyse de la distribution squelettique est un aspect important de l'analyse taphonomique car, selon le facteur considéré (e.g., proie, prédateur, taille, âge), elle apporte des indications quant au mode d'occupation (carnivore vs. naturel vs. anthropique) et à la caractérisation des interactions (Fosse 1994 ; Fosse et al. 2001 ; Fourvel 2012 ; Fourvel et al. 2014a). Cette étude tient compte de la fracturation osseuse. Le taux de fracturation sur os frais (sensu Villa \& Mahieu 1991) a été observé ainsi que la présence de traces de consommation. Pour autant, si le bord de fracture n'a pu être caractérisé ou qu'aucune trace n'est observable cela ne signifie pas pour autant que l'os n'a pas été exploité. 
La fragmentation des os longs est analysée à travers les fréquences de représentation de chaque portion osseuse. Six portions (en plus des os complets ou partiellement complet) ont été reconnues : les extrémités (proximale ou distale) seules, les extrémités associées à une portion diaphysaire, les cylindres ou les éclats diaphysaires.

Les différences d'intensité de consommation peuvent être observées à partir de l'enregistrement des différents types de traces de carnivores observables. La typologie employée s'inspire des travaux les plus couramment employés (Sutcliffe 1970 ; Binford 1981 ; Brain 1981) et tels que synthétisés lors de travaux antérieurs (Fourvel 2012 ; Fourvel et al. 2014a).

Par ailleurs, des analyses polliniques ont été entreprises par l'une d'entre nous (JA) en vue de décrire le cadre paléoenvironnemental. Les sédiments du réseau Salomé ne se prêtent ni au dépôt du pollen ni à sa conservation satisfaisante. Les coprolithes restent alors le seul moyen d'obtenir des informations fiables sur le paléoenvironnement (absorption quotidienne des grains de pollen et des spores, résistant à l'action des sucs digestifs, et se retrouvant dans les excréments). Le contenu reflète nécessairement ce qu'un individu a absorbé sur un trajet précis, dans un cadre limité (local ou régional) et, par conséquent, les grains de pollen appartenant aux végétaux présents dans l'aire de déplacement de l'animal. Même s'il se trouve en faible quantité il donne donc sur l'environnement des indications que l'on ne peut négliger (Argant et Philippe 2011 ; Argant 2014). Quatre coprolithes d'hyène du réseau Salomé ont été sélectionnés pour une analyse pollinique. Le traitement des échantillons s'est fait suivant la méthode habituelle de concentration en liqueur dense $(d=2)$ utilisée pour les sédiments minéraux, après brossage énergique de la surface (AC 99 et AC 100) pour éliminer le sédiment encaissant adhérant éventuellement à la surface :

- décarbonatation par l'acide chlorhydrique

- désilicification par l'acide fluorhydrique (40\% à froid)

- traitement par la potasse à chaud (10 min.) pour éliminer la matière organique

- concentration en liqueur dense $(d=2)$ après mixage et centrifugation.

- montage du culot dans la glycérine.

Enfin, une série de datation radiocarbone $\left({ }^{14} \mathrm{C}\right.$ AMS) a été entreprise. Cinq échantillons osseux ont été sélectionnés pour datation, représentant trois taxons différents : I'hyène des cavernes (une portion de fémur $n^{\circ} 58$ et un métapode $n^{\circ} 497$ ), l'ours des cavernes (un fragment d'humérus $n^{\circ} 10$ et un métapode $n^{\circ} 411$ ) et le bouquetin (la portion de scapula du Locus F). Cet ensemble de datations apparaissait d'autant plus important pour l'hyène, puisque nous ne disposions au moment de l'étude d'aucune date directe sur cette espèce pour cette région (Fourvel, Lateur et Philippe 2016).

\begin{tabular}{|c|c|c|c|c|c|c|}
\hline \multicolumn{7}{|c|}{ Faune in situ } \\
\hline Espèce & NR & $\%$ NR & NME & \%NME & NMI & \%NMI \\
\hline Ursus spelaeus & 392 & 67 & 328 & 71 & 7 & 35 \\
\hline Crocuta crocuta spelaea & $140\left(+21^{\star}\right)$ & 24 & $121\left(+19^{*}\right)$ & 26 & 9 & 45 \\
\hline cf. Crocuta & 6 & 1 & 6 & 1 & - & - \\
\hline Panthera (leo) spelaea & 1 & $<1$ & 1 & $<1$ & 1 & 5 \\
\hline Vulpes vulpes & - & - & - & - & - & - \\
\hline Carnivora & 18 & 3 & - & - & - & - \\
\hline Capra ibex & 2 & $<1$ & 2 & $<1$ & 2 & 10 \\
\hline Bovidae & 1 & $<1$ & 1 & $<1$ & - & - \\
\hline Lagomorpha & 2 & $<1$ & 2 & $<1$ & 1 & 5 \\
\hline Indéterminé & 17 & 3 & - & - & - & - \\
\hline Total & $581\left(+21^{*}\right)$ & 100 & $461\left(+19^{*}\right)$ & 100 & 20 & 100 \\
\hline \multicolumn{7}{|c|}{ Faune Hors-Stratigraphie } \\
\hline Espèce & NR & $\%$ NR & NME & \%NME & NMI & \%NMI \\
\hline Ursus spelaeus & 35 & 67 & 33 & 66 & 2 & 29 \\
\hline Crocuta crocuta spelaea & $9\left(+4^{*}\right)$ & 25 & $9\left(+4^{*}\right)$ & 26 & 3 & 43 \\
\hline cf. Crocuta & - & - & - & - & - & - \\
\hline Panthera (leo) spelaea & - & - & - & - & - & - \\
\hline Vulpes vulpes & 1 & 2 & 1 & 2 & 1 & 14 \\
\hline Carnivora & - & - & - & - & - & - \\
\hline Capra ibex & 3 & 6 & 3 & 6 & 1 & 14 \\
\hline Bovidae & - & - & - & - & - & - \\
\hline Lagomorpha & - & - & - & - & - & - \\
\hline Indéterminé & - & - & - & - & - & - \\
\hline Total & $48\left(+4^{*}\right)$ & 100 & $46\left(+4^{*}\right)$ & 100 & 7 & 100 \\
\hline
\end{tabular}

Tableau 1 - Spectre faunique (NR, $\% N R, N M E, \% N M E, N M I, \% N M I)$ du matériel osseux mis au jour en stratigraphie et hors contexte. ${ }^{*}=$ Coprolithes.

Table 1 - Faunal spectrum (NISP, $\%$ NISP, MNE, \%MNE, MNI, $\% M N I)$ from the palaeontological deposit and the reworked loci. ${ }^{*}=$ Coprolithes. 


\section{4 - Résultats}

\section{1 - Examen paléontologique}

Famille Felidae Fischer de Waldheim, 1817

Sous-famille Pantherinae Pocock, 1917

Genre Panthera Oken, 1816

Panthera (Leo) spelaea (Goldfüss, 1810)

L'examen du matériel osseux a permis la détermination d'un grand Félidé : une phalange 1 aux traits typiquement félins (compression transversale de l'extrémité distale formant une gorge étroite, courbure dorso-palmaire accentuée) et à la taille importante ( $\mathrm{GL}=57,9 \mathrm{~mm}$, DTm $=$ $19,1 \mathrm{~mm}$ ) plaident en faveur du lion des cavernes Panthera (leo) spelaea.

\section{Famille Hyaenidae Gray, 1821 \\ Genre Crocuta Kraup 1828 \\ Crocuta crocuta spelaea (Goldfüss, 1823)}

Le genre Crocuta Kaup, 1828 se distingue des autres genres par d'importantes différences morphologiques et rassemble la plus grande diversité (sous-)spécifique d'hyènes plio-pléistocènes (Werdelin \& Solounias 1991 ; Turner, Anton et Werdelin 2008). La lignée évolutive européenne de Crocuta se caractérise par d'importantes fluctuations de taille rapportées aux variations climatiques (Kurtén 1958 ; Kurtén \& Poulianos 1977 ; Klein \& Scott 1989). Les restes osseux et dentaires d'hyène représentent près de $25 \%$ du NRT. Si à première vue l'attribution du matériel à la sous-espèce $C$. crocuta spelaea ne fait aucun doute, l'analyse paléontologique est l'occasion de caractériser sa variabilité morphométrique.

\section{- Squelette crânien}

Deux fragments crâniens ont été rapportés à l'hyène : une portion associant ptérygoïde et fosse mandibulaire $\left(n^{\circ} 128\right)$ et un os nasal gauche ( $\left.n^{\circ} 374\right)$. Toutes les dents jugales supérieures sont représentées (dents déciduales et dents définitives). Les données odontométriques sont figurées dans le tableau 2.
La D2 ( $\left.n^{\circ} 252\right)$ est une dent de morphologie conique et unicuspide. La D3 ( $\left.n^{\circ} 410\right)$ présente un protocône prononcé reposant sur une imposante racine. Cette cuspide est située à l'arrière de deux petits denticules. Le métastyle est court, ne dépassant guère le tiers du DMD total de la dent. La canine $n^{\circ} 154$ fait partie des dents les plus robustes reconnues pour l'OIS 3. Deux P1, dent unicuspide de forme sub-circulaire, ont été déterminées. Une arête saillante traverse la couronne selon un axe mésio-distal et la base de la couronne est marquée par un fort bourrelet cingulaire.

Cinq P2 ont pu être observées. Cette dent est morphologiquement proche de son homologue inférieur p2, présentant un protocône flanqué antérieurement d'une cuspide accessoire. La dent est traversée d'une arête saillante qui, dans la partie antérieure, se poursuit vers la face linguale pour aboutir sur un cingulum très proéminent bordant l'ensemble de la face interne.

De forme conique et haute, la P3 participe au cassage des os. Elle se distingue de la p3 par la présence d'une arête saillante courant le long de la dent et aboutissant, distalement, en face linguale sur un bourrelet cingulaire important. Quatre dents répondent à cette description au réseau Salomé. Les variations de taille de l'hyène au cours du Pléistocène se répercutent sur la P4 : l'allongement progressive du métastyle traduit cette influence (Kurtén 1956 ; Kurtén \& Poulianos 1977 ; Werdelin \& Solounias 1991 ; Baryshnikov 1999). La comparaison des valeurs des $\mathrm{P} 4$ du réseau Salomé $(\mathrm{NR}=6)$ avec d'autres populations du Pléistocène moyen et supérieur s'accorde avec la tendance générale (tabl. 3).

Trois hémi-mandibules presque complètes, rapportées à deux adultes et un juvénile, ont été mises au jour (fig. 4). Les deux mandibules adultes présentent l'ensemble des caractéristiques du genre Crocuta : concavité du bord inférieure de la branche horizontale avec développement maximal sous la $\mathrm{m} 1$; la fosse massétérique débute sous la $\mathrm{m} 1$; enfin la $\mathrm{m} 1$ est relativement fine avec un trigonide développé et un talonide réduit.

\begin{tabular}{lcccccc}
\hline \multicolumn{7}{c}{ Jugales supérieures } \\
\hline Rang & N & Min-Max & Moy. & N & Min-Max & Moy. \\
\hline C & 2 & $13,2-14,4$ & 13,8 & 2 & $16,2-19,1$ & 17,65 \\
P1 & 2 & $6,2-7,4$ & 6,8 & 2 & $7,6-8,0$ & 7,8 \\
P2 & 5 & $13,8-14,8$ & 14,3 & 5 & $18,4-19,2$ & 18,9 \\
P3 & 4 & $17,4-19,5$ & 18,7 & 4 & $24,8-25,3$ & 25,2 \\
\hline D2 & 1 & - & 7 & 1 & - & 11,4 \\
D3 & 1 & - & 15 & 1 & - & 22,8 \\
\hline \multicolumn{7}{c}{ Jugales inférieures } \\
\hline Rang & $\mathbf{N}$ & Min-Max & Moy. & $\mathbf{N}$ & Min-Max & Moy. \\
\hline C & 2 & $14,5-15,0$ & 14,75 & 2 & $18,0-18,0$ & 18 \\
p2 & 1 & - & 14 & 1 & - & 18,3 \\
p4 & 3 & $14,2-15,8$ & 14,8 & 3 & $24,4-24,8$ & 24,6 \\
\hline
\end{tabular}

Tableau 2 - Odontométrie des dents jugales supérieures et inférieures d'hyène des cavernes du réseau Salomé. Mesures en $\mathrm{mm}$.

Table 2 - Measurements of the cave hyena upper and lower teeth from Réseau Salomé. Mesures in $\mathrm{mm}$. 


\begin{tabular}{|c|c|c|c|c|c|c|c|c|}
\hline & Site & Salomé & Fouvent & Conives & Baume-Longue & Châtillon & Lunel-Viel 1 & Mosbach \\
\hline & Période & OIS3 ? & OIS3 & OIS3 & OIS3 & 250ka & 350ka & P. moy. \\
\hline & $\mathbf{N}$ & 4 & 19 & 13 & 9 & 8 & 48 & 2 \\
\hline \multirow[t]{3}{*}{ DVL } & Min-Max & $22-25$ & $19-23$ & $20-23$ & $20,6-26$ & $21-23$ & $17,7-22,4$ & $18,7-19,5$ \\
\hline & Moy & 22,9 & 21,6 & 21,4 & 23,2 & 21,8 & 20,3 & 19,1 \\
\hline & $\mathbf{N}$ & 4 & 16 & 13 & 12 & 8 & 48 & 2 \\
\hline \multirow[t]{2}{*}{ DMD } & Min-Max & $39,6-41,3$ & $35,5-42$ & $38-42,7$ & $39,1-42,7$ & $39,6-43,5$ & $34,2-39,7$ & $41,6-42,9$ \\
\hline & Moy & 40,2 & 39,7 & 39,8 & 41,2 & 41 & 37 & 42,3 \\
\hline \multirow{3}{*}{$\begin{array}{l}\text { DMD } \\
\text { métas }\end{array}$} & $\mathbf{N}$ & 5 & 19 & 14 & 10 & 3 & 22 & 2 \\
\hline & Min-Max & $17,2-19$ & $15-20$ & $16,3-18,4$ & $16,5-19,1$ & $16-16,8$ & $11,4-16,8$ & $17-18,3$ \\
\hline & Moy & 18,1 & 18 & 17,4 & 17,9 & 16,5 & 15,4 & 17,7 \\
\hline \multirow{4}{*}{$\begin{array}{l}\text { DMD } \\
\text { parac }\end{array}$} & $\mathbf{N}$ & 4 & & 13 & 9 & 3 & 23 & 2 \\
\hline & Min-Max & $13-15,5$ & & $14,8-17,5$ & $13,3-14,8$ & $14-15,5$ & $12,4-18,9$ & $13,6-15,3$ \\
\hline & Moy & 13,9 & & 15,5 & 14,2 & 14,8 & 13,7 & 14,5 \\
\hline & Source & 1 & 2 & 2 & 2 & 3 & 4 & 5 \\
\hline
\end{tabular}

Tableau 3 - Odontométrie comparée des quatrièmes prémolaires supérieures $(P 4)$ d'hyène des cavernes. Mesures en mm. Sources : 1 - Cette étude ; 2- Fourvel 2012 ; 3- Chauviré 1962 ; Cardoso 1993 ; 4- Bonifay 1971 ; Cardoso 1993 ; Fosse com. pers. ; 5 - Kurtén 1962, 1972.

Table 3 - Hyena upper carnassial P4 measurements. Mesures in mm. References : 1- This study ; 2- Fourvel 2012 ; $3-$ Chauviré 1962 ; Cardoso 1993 ; 4- Bonifay 1971 ; Cardoso 1993 ; Fosse com. pers. ; 5- Kurtén 1962, 1972."

Les données odontométriques des dents jugales inférieures sont figurées dans le tableau 2. Une p2 a été reconnue : dent relativement longue, protocône flanqué d'un denticule antérieur, talon large formé par le denticule postérieur, absence de cingulum en face linguale. Trois $p 4$, dent longue composée de trois cuspides marquées (paraconide, protoconide, hypoconide), ont été mises au jour au réseau Salomé. La m1 de Crocuta est composée d'un trigonide associant un paraconide et un protoconide (ce dernier plus long que le premier, est parfois flanqué d'un métaconide) et d'un talonide extrêmement réduit. Les pièces du réseau Salomé (NR=4) sont comparables aux populations contemporaines (tabl. 4). L'évolution de la carnassière de l'hyène des cavernes sensu lato est marquée par un accroissement des parties sécantes depuis le Pléistocène moyen jusqu'au stade isotopique 3 (Kurtén 1963 ; Klein \& Scott 1989). Les $\mathrm{m} 1$ du réseau Salomé, de forte taille, s'intègrent tout à fait dans ce scénario.

\section{- Squelette post-crânien}

La diversité des éléments post-crânien du réseau Salomé $(\mathrm{NR}=90)$ autorise une description de ces pièces encore trop peu étudiées. Même si l'assemblage présente de nombreux restes du squelette axial, des blocs carpiens et tarsiens, nous nous arrêterons ici à l'examen des os longs et métapodes. Ces autres éléments feront l'objet d'une analyse détaillée ultérieure. L'ensemble des données ostéométriques des os longs est figurée dans le tableau 5 (membres antérieur et postérieur).

Trois humérus ont été identifiés. C'est un os assez court et massif dont l'extrémité proximale porte un tubercule majeur développé et l'extrémité distale présente un foramen supratrochléaire. La diaphyse est transversalement comprimée et s'élargit progressivement antéro-postérieurement au tiers de sa longueur et ce jusqu'à l'extrémité proximale. Le radius se caractérise par une diaphyse longue présentant une courbure en S (Bonifay 1971). Les six radius identifiés présentent des proportions variables. La différence des longueurs (GL) entre les $n^{\circ} 92$ et $n^{\circ} 487$ suggère un éventuel dimorphisme sexuel (fig. 5).

Les métacarpiens d'hyène ont fait l'objet de peu de descriptions. II s'agit d'os assez rectilignes. L'extrémité distale présente un profil rectangulaire en face dorsale. La massivité et la robustesse de ces éléments les distinguent des Canidés généralement plus graciles. Dix métacarpiens ont été reconnus au réseau Salomé. Trois métacarpiens 3 ont été déterminés avec une différence marquée entre $n^{\circ}$ 198 et $n^{\circ} 597$ posant la question de cette variabilité et d'un potentiel dimorphisme sexuel. Les valeurs des métacarpiens 4 attestent bien de la robustesse des hyènes du réseau Salomé avec des longueurs (GL) supérieures aux hyènes würmiennes de la Baume-Longue (presque $90 \mathrm{~mm}$ ). La portion proximale droite de métacarpe 5 traduit aussi cette massivité avec des proportions Bp/Dp importantes.

Le fémur se caractérise par une tête articulaire ronde, nettement dissociée de l'extrémité proximale, une diaphyse de section subcirculaire avec arête saillante sur le bord postéro-externe formant un angle droit dans la partie supérieure, et une extrémité distale marquée par la dissymétrie des lèvres de la trochlée. La portion distale $n^{\circ} 200$ suggère un animal robuste avec une diaphyse large et une extrémité aux proportions importantes. Deux tibias gauches complets ont été mis au jour. Cet os court et massif montre un plateau tibial peu large et donnant naissance à une crête saillante. La différence flagrante entre les deux pièces du réseau Salomé pose question (20mm de différence GL ; $5 \mathrm{~mm}$ de différence $\mathrm{Dp}$ ). Le problème reste de savoir dans quelle mesure ces différences reflètent la variabilité propre à l'hyène tachetée pléistocène ou si elles peuvent être le résultat d'un dimorphisme sexuel (fig. 6). 

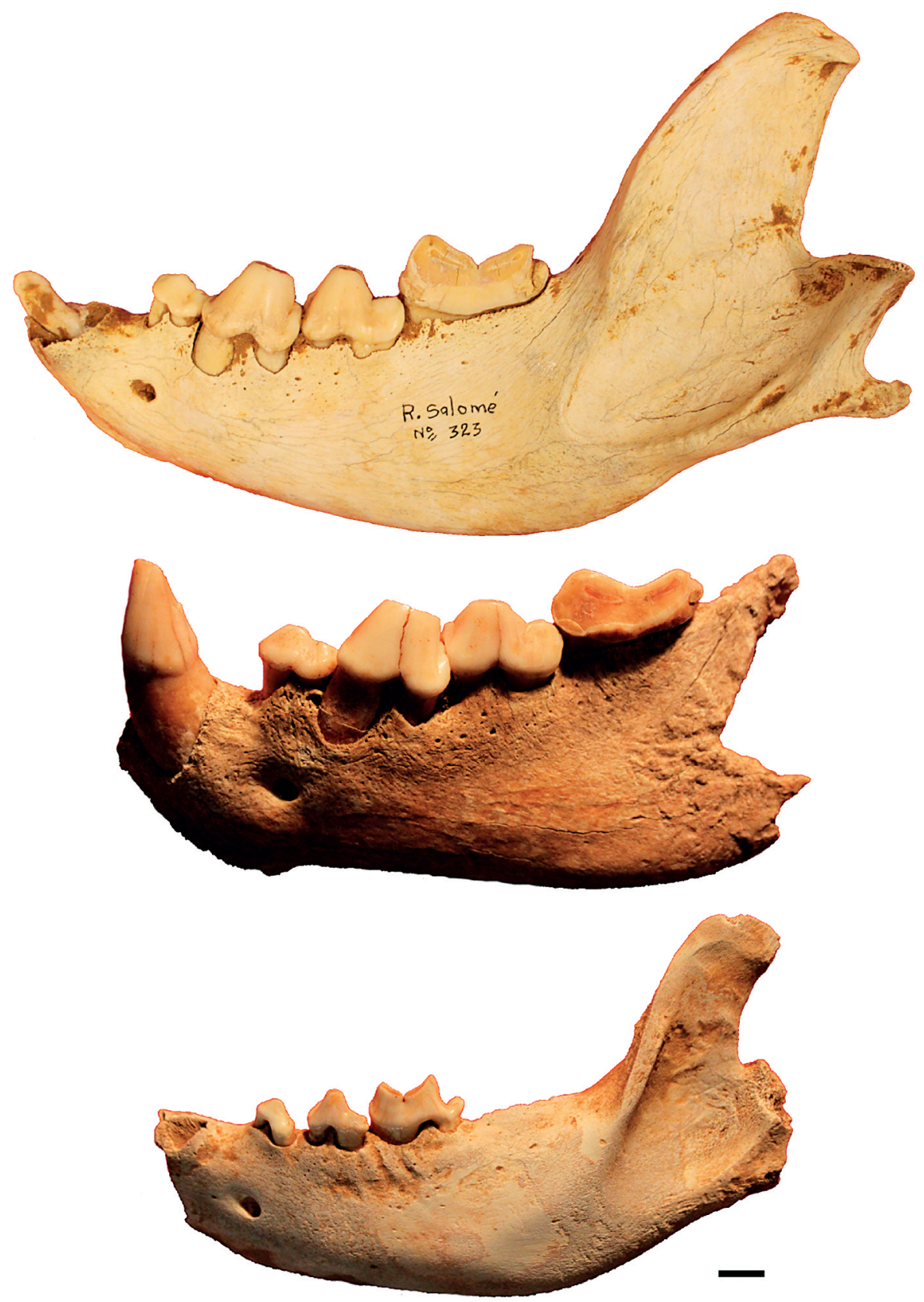

Figure 4 - Mandibules d'hyène du réseau Salomé. De haut en bas : $n^{\circ} 323$, Locus J, Locus K.

Photographies JBF. Echelle : $10 \mathrm{~mm}$.

Figure 4 - Hyena mandibles from Réseau Salomé. From up to down : $n^{\circ} 323$, Locus J, Locus K. Pictures JBF. Bar scale: $10 \mathrm{~mm}$.

Onze métatarsiens d'hyène ont été identifiés dont trois métatarsiens 2, un métatarsien 3 droit complet, trois métatarsiens 4 complets et trois métatarsiens 5 . Les métatarsiens 2 à $5 n^{\circ} 482, n^{\circ} 466, n^{\circ} 467$ et $n^{\circ} 481$ présentent tous une légère exostose et appartiennent au même individu.

\section{Famille Ursidae Fischer de Waldheim, 1817 Genre Ursus Linnaeus, 1758 Ursus spelaeus Rosenmüller \& Heinroth, 1794}

Les Ursidés sont abondamment représentés dans les cavités ardéchoises, en particulier l'ours des cavernes,
$U$. spelaeus. Le réseau Salomé est un nouvel exemple de l'importance de ce taxon dans les contextes karstiques des gorges de l'Ardèche. Plus de 400 vestiges lui sont rapportés (392 en stratigraphie et 35 hors-contexte), soit plus de $65 \%$ du NRT. L'ours des cavernes étant une espèce largement connue, nous ne donnerons ici qu'un aperçu général et ceci d'autant plus que peu d'ossements sont suffisamment complets pour donner lieu à des mensurations. En effet, une bonne partie du matériel osseux a souffert des actes de prédation (cf. 4.3 Analyse taphonomique). 


\begin{tabular}{|c|c|c|c|c|c|c|c|}
\hline \multicolumn{2}{|c|}{ Site } & Salomé & Fouvent & Conives & Peyre & Châtillon & Lunel-Viel 1 \\
\hline \multicolumn{2}{|c|}{ Période } & OIS3 ? & OIS3 & OIS3 & OIS5e? & 250ka & 350ka \\
\hline & $\mathbf{N}$ & 4 & 46 & 19 & 9 & 12 & 33 \\
\hline \multirow[t]{3}{*}{ DVL } & Min-Max & $13-15$ & $12-14,5$ & $12,6-16$ & $12,3-15$ & $12,3-15,5$ & $11,2-14$ \\
\hline & Moy & 13,9 & 13,4 & 14,1 & 13,4 & 13,6 & 12,6 \\
\hline & $\mathbf{N}$ & 4 & 42 & 18 & 8 & 12 & 34 \\
\hline \multirow[t]{2}{*}{ DMD } & Min-Max & $31,2-32,9$ & $27,5-34$ & $29-36,5$ & $28-33,4$ & $29,5-34,4$ & $26-33$ \\
\hline & Moy & 32,2 & 31,7 & 32 & 30,5 & 31,2 & 29,4 \\
\hline \multirow{4}{*}{$\begin{array}{l}\text { DMD } \\
\text { trigonide }\end{array}$} & $\mathbf{N}$ & 4 & 41 & 15 & 9 & 1 & 17 \\
\hline & Min-Max & $26,5-27,9$ & $23,7-29$ & $24-30,5$ & $23-28$ & 24,8 & $22,8-28,7$ \\
\hline & Moy & 27,2 & 27 & 27,6 & 25,3 & & 26,4 \\
\hline & Source & 1 & 2 & 2 & 2 & 3 & 4 \\
\hline
\end{tabular}

Tableau 4 - Odontométrie comparée des premières molaires inférieures (m1) d'hyène des cavernes. Mesures en $\mathrm{mm}$. Sources : voir tableau 3.

Table 4 - Hyena lower carnassial m1 measurements. Mesures in mm. References : see table 3."

\section{- Squelette crânien}

Le squelette crânien est représenté par 98 éléments (dont 65 dents isolées). Aucun crâne (sub-)complet n'a été recueilli. Notons la présence d'un maxillaire droit d'un adulte avec les dents jugales en place montrant des traces d'usure amorcée, une hémimandibule gauche cassée au départ de la branche montante d'un jeune adulte avec sa canine et les $\mathrm{m} 2$ et $\mathrm{m} 3$ sans traces d'usure, une hémimandibule droite d'un animal âgé cassée au niveau de la p4 et avec une partie de la branche montante possédant les trois molaires nettement usées, ainsi qu'un important fragment d'hémimandibule droite d'un sub-adulte muni de la p4 et de la m3 encore en partie incluse dans la branche montante (fig. 7). Quarante-trois dents jugales, 8 canines et 14 incisives ont été recueillies. Le reste correspond à de petits fragments de crânes.

\section{- Squelette post-crânien}

Trois cents vingt-neuf éléments post-crâniens ont été mis au jour. Aucun os long n'est complet ; pratiquement tous ont leurs extrémités endommagées. Tout au plus on peut noter une fibula presque intacte et quelques diaphyses de juvéniles ou de subadultes sans traces de prédation. Comme c'est généralement le cas, les os des autopodes ont moins souffert de la prédation et sont pour la plupart en meilleur état. Le nombre minimum d'individus peut être estimé à 7.

\section{2 - Structure de mortalité}

\subsection{1 - La population d'hyène}

Trente quatre éléments crâniens (maxillaires et mandibules) et dentaires ont pu être rapportés à l'une des classes d'âge définies. Les résultats obtenus sur la base du Nombre de Restes (NR) et sur le Nombre Minimum d'Individus (NMI) diffèrent sensiblement (tabl. 6). La structure de mortalité est caractérisée par deux juvéniles (stade 1) et deux adultes (stade 3). Les adultes âgés (stade 4) sont les plus fréquents $(\mathrm{NMI}=3)$ et les séniles (stade 5) et jeunes adultes (stade 2) sont les moins fréquents (un individu par stade). Notons cependant que les classes d'âge correspondant aux animaux adultes et plus sont les mieux représentées (stades 3-5: NMl=6). Les 95 restes post-crâniens inclus dans cette étude correspondent à un total de 5 individus. Sur la base de ces restes, les individus adultes et plus âgés dominent l'assemblage (tabl. 6).

Cependant, la population étant relativement faible $(\mathrm{NMI}=9$ pour les éléments dentaires et $\mathrm{NMI}=5$ pour les éléments post-crâniens), ces résultats doivent être considérés avec toutes les réserves qui s'imposent.

\subsection{2 - La population ursine}

Sept individus (NMIc) ont pu être décomptés à partir du matériel dentaire et post-crânien ( 7 fémurs droits, 7 humérus gauches) dont un juvénile, quatre adultes et un adulte âgé. $\mathrm{Si}$ le matériel dentaire suggère une assez faible représentation des juvéniles, les restes post-crâniens temporisent nettement cette structure de population. En effet, parmi les 329 éléments post-crâniens, 64 (20\% du NRD ours) correspondent à des juvéniles dont une diaphyse d'humérus de très petite taille et un fragment de vertèbre attestant de la présence d'un ourson nouveau-né (fig. 8). Malgré tout, bien peu des éléments post-crâniens peuvent être classés de façon précise dans l'un des quatre stades ontogéniques classiques (néonataux, juvéniles, sub-adultes et adultes y compris séniles). Nous avons toutefois tenté de procéder à un tel classement à partir des os longs et des métapodes. Les os longs étant souvent réduits à l'état de fragments en raison de l'importance de la prédation qui se traduit presque toujours par la disparition des épiphyses, n'ont pu être exploités pour la présente étude que 4 humérus, 5 radius, 3 ulnas, 4 fémurs, 3 tibias et une seule fibula. Concernant les métapodes, si la liste exhaustive du matériel osseux en compte 35 , il ne s'agit parfois que d'épiphyses. La logique veut donc que l'on ne prenne en compte que les corps des métapodes recensés, soit 23 spécimens car, sinon, le stade sub-adulte serait surestimé. 


\begin{tabular}{|c|c|c|c|c|c|c|c|}
\hline $\mathbf{N}^{\circ}$ & Elément & $\mathbf{G L}$ & $\mathrm{Bp}$ & $D p$ & SD & Bd & Dd \\
\hline $\mathrm{n}^{\circ} 294$ & Humérus & 221,7 & 57,9 & 70,8 & 20,9 & 55,2 & 41,9 \\
\hline$n^{\circ} 266$ & Humérus & - & - & - & 21,6 & - & - \\
\hline$n^{\circ} 92$ & Radius & 209,5 & 31,1 & 20,4 & 20,2 & 42,9 & 24,9 \\
\hline$n^{\circ} 487$ & Radius & 218 & 30,7 & 20,5 & 22,3 & 42,6 & 25,57 \\
\hline$n^{\circ} 32$ & Radius & - & 31 & 19,7 & 21,9 & - & - \\
\hline$n^{\circ} 102$ & Radius & - & - & 21,8 & - & - & - \\
\hline$n^{\circ} 576$ & Radius & - & 30,6 & 20,6 & 20,4 & - & - \\
\hline$n^{\circ} 556$ & Radius & - & - & - & - & 42,9 & 25,8 \\
\hline$n^{\circ} 156$ & Mtc $2^{(1)}$ & - & 16 & 18,1 & 12 & - & - \\
\hline $\mathrm{n}^{\circ} 198$ & Mtc 3 & 95 & 15 & - & 12,3 & 17 & 16 \\
\hline$n^{\circ} 597$ & Mtc 3 & 83,2 & 15 & 19 & 11,4 & 15 & 14,8 \\
\hline$n^{\circ} 112$ & Mtc $3^{(1)}$ & - & 16 & 20,6 & 12 & - & - \\
\hline$n^{\circ} 501$ & Mtc $4^{(1)}$ & - & 15 & 20 & 11,5 & - & - \\
\hline$n^{\circ} 149$ & Mtc 4 & 89,5 & 14,7 & - & 12 & 16 & 16 \\
\hline$n^{\circ} 504$ & Mtc 4 & 89,2 & 13,5 & 17 & 10 & 14,5 & 15 \\
\hline $\mathrm{n}^{\circ} 504$ & Mtc 4 * & $(72,3)$ & $(13,0)$ & $(16,0)$ & $(9,0)$ & - & - \\
\hline$n^{\circ} 378$ & Mtc $5^{(1)}$ & - & 19,4 & 19 & 12,7 & - & - \\
\hline $\mathrm{n}^{\circ} 200$ & Fémur & - & - & - & 24,1 & 57,4 & 53,4 \\
\hline$n^{\circ} 373$ & Fémur & - & - & - & 26 & - & - \\
\hline$n^{\circ} 193$ & Fémur & - & - & - & 21 & - & - \\
\hline$n^{\circ} 190$ & Fémur & - & - & - & 22 & - & - \\
\hline$n^{\circ} 352$ & Fémur * & - & - & 31,7 & - & - & - \\
\hline $\mathrm{n}^{\circ} 322$ & Tibia & 183,2 & 51,6 & 55,7 & 23 & 38,1 & 25,5 \\
\hline$n^{\circ} 147$ & Tibia & 203,5 & 53,3 & 60,5 & 20,9 & 39,5 & 26 \\
\hline$n^{\circ} 482$ & Mtt $2^{(2)}$ & 74,5 & 12 & 18,2 & 12,8 & 15,2 & 13,8 \\
\hline$n^{\circ} 572$ & Mtt 2 & - & 11 & 18 & 10,5 & - & - \\
\hline $\mathrm{n}^{\circ} 479$ & Mtt 2 & 76 & 11,5 & 19 & 11,5 & 15,2 & 14,3 \\
\hline $\mathrm{n}^{\circ} 466$ & Mtt $3^{(2)}$ & 83 & 16 & 21,4 & 11,8 & 15,8 & 14 \\
\hline$n^{\circ} 467$ & Mtt $4^{(2)}$ & 81 & 11,5 & 17,5 & 10 & 13,2 & 13 \\
\hline$n^{\circ} 402$ & Mtt 4 & 80 & 10,5 & 18,2 & 11 & 14 & 13 \\
\hline$n^{\circ} 158$ & Mtt 4 & 83 & 11 & 18,5 & 11,2 & 14,2 & 13,8 \\
\hline$n^{\circ} 481$ & Mtt $5^{(2)}$ & 62 & 12,4 & 17,7 & 8,5 & 12,1 & 12 \\
\hline$n^{\circ} 206$ & Mtt 5 & 67,8 & 11,1 & 17,5 & 8,5 & 11 & 12 \\
\hline $\mathrm{n}^{\circ} 206$ & Mtt 5 & - & 13 & 18,2 & 8,5 & - & - \\
\hline
\end{tabular}

Tableau 5 - Ostéométrie des os longs des membres antérieur et postérieur d'hyène des cavernes. Mesures en $\mathrm{mm} .(x)=$ éléments appartenant à un même individu ; *= juvénile/subadulte. $(x)=$ mesure approximative.

Table 5 - Hyena fore limb and hind limb bones measurement. Mesures in $\mathrm{mm} .(\mathrm{x})=$ elements related to the same individual.

${ }^{*}=$ juvenile/sub-adult.

$(x)=$ estimated measures.
Le tableau 7 donne les résultats obtenus. On notera qu'il n'y a pratiquement pas de vestiges de néonataux alors que les autres stades sont répartis de façon assez homogène, avec cependant un pourcentage bien plus élevé de juvéniles représentés par des os longs (50\% à partir des os longs pour seulement $8,7 \%$ à partir des métapodes).

\section{3 - Analyse taphonomique}

\subsection{1 - Représentation squelettique}

L'analyse de la représentation squelettique inclut un total de 200 restes d'ours et 124 d'hyène (tabl. 8). À partir de l'examen de la distribution squelettique des hyènes, on note que l'ensemble des éléments anatomiques sont bel et bien présents (crâniens (crâne + mandibules), axiaux (vertèbres + ceinture scapulaire + ceinture pelvienne) et appendiculaires (membres antérieurs + membres postérieurs). Si certaines portions anatomiques semblent déficitaires (e.g., côtes et vertèbres), il n'en reste pas moins que la distribution suggère que les caracasses étaient à l'origine complètes au sein même de la cavité. La représentation des différents éléments squelettiques d'ours atteste, tout comme l'hyène, que l'ensemble des portions anatomiques est présent. Par ailleurs, la comparaison du NR par élément anatomique avec le NME associé témoigne du faible taux de fragmentation (cf. infra) et de la présence d'ossements souvent complets. Ceci est particulièrement vrai pour l'hyène des cavernes.

\subsection{2 - Fragmentation et morphotypes osseux}

Les résultats de la représentation de chaque portion osseuse pour les ours et les hyènes sont réunis dans le tableau 9. Malgré un échantillon faible (34 NRD), et en dépit de l'absence régulière des extrémités (rongées), la fragmentation osseuse des restes d'ours est peu intense. En effet, si la majeure partie des os est consommée (cylindre dominant pour chaque os excepté I'humérus), la séquence atteint rarement son niveau extrême. Une fois les extrémités consommées, l'os est abandonné à l'état de cylindre. Notons cependant une fragmentation plus importante des humérus et des fémurs pour lesquels des éclats diaphysaires formés lorsque l'os possédait encore ses propriétés élastiques ont été identifiés (respectivement 4 NRD-33 \% et 3 NRD-27 \%). La présence de ces éclats peut apparaître comme la signature taphonomique de 

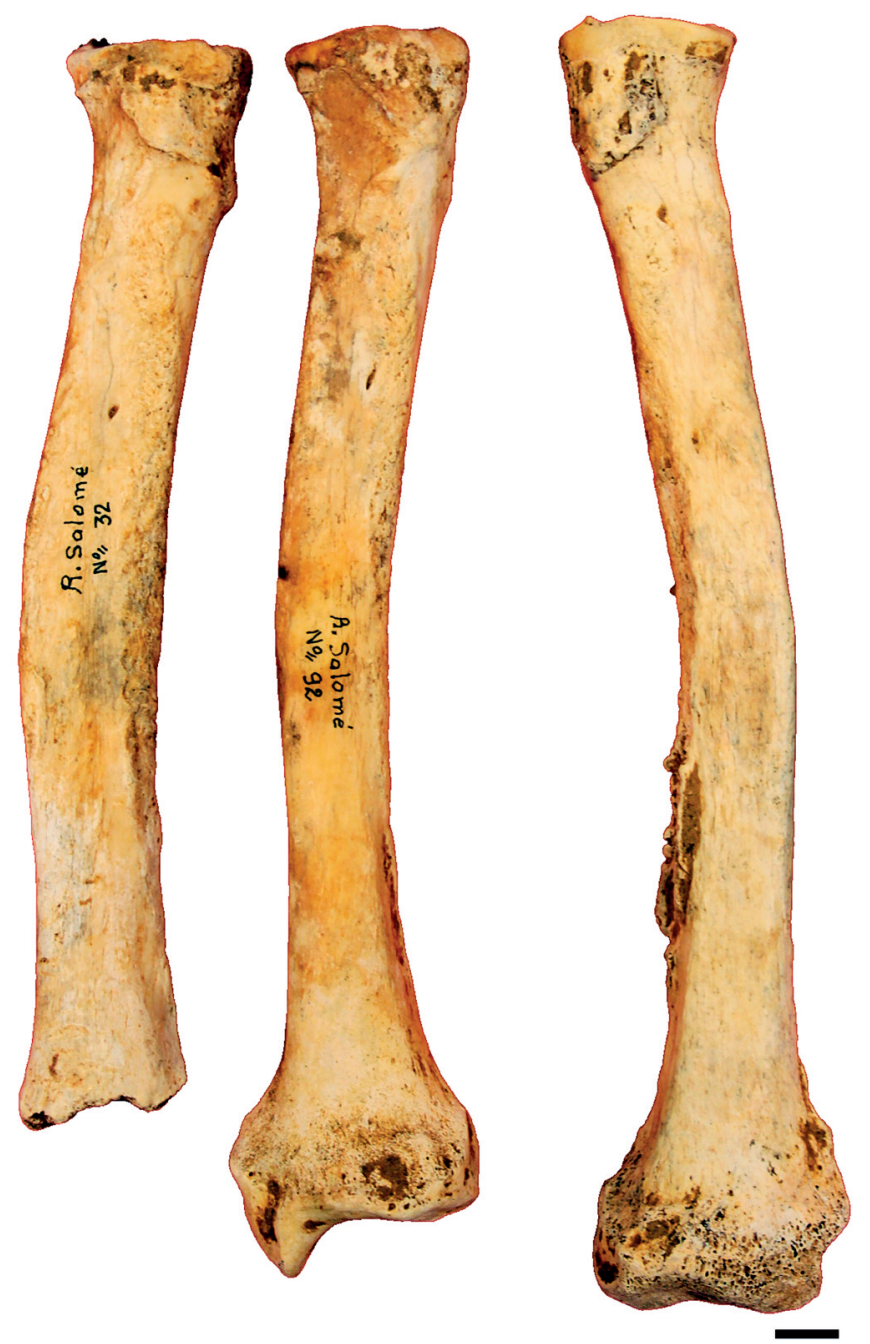

Figure 5 - Radius d'hyène du réseau Salomé. De gauche à droite : $n^{\circ} 32, n^{\circ}$ 92, $n^{\circ}$ 487. Photographies JBF. Echelle : $10 \mathrm{~mm}$.

Figure 5 - Hyena radius from Réseau Salomé. From left to right : $n^{\circ} 32, n^{\circ} 92, n^{\circ} 487$. Pictures JBF. Bar scale : $10 \mathrm{~mm}$.

l'hyène, ce prédateur disposant d'une denture adaptée à la fragmentation osseuse au contraire de l'ours. Par ailleurs, notons que la consommation (fragmentation, traces) des juvéniles est attestée par la présence de plusieurs épiphyses conservées (8 NRD pour les oursons ; 2 NRD pour les hyénons) et présentant les stigmates caractéristiques (e.g., traces de dents, encoches).

La distribution des restes d'hyène semble relativement similaire à celle de l'ours. Cependant, l'absence d'éclats diaphysaires suggère un taux de fragmentation moins fort.

\subsection{3 - Traces de consommation}

Au réseau Salomé, huit types de traces ont été observés (tabl. 10 - fig. 9). Malgré un nombre de stigmates assez faible ( $n$ total $=41 ; n$ ours $=23 ; n$ hyène $=18$ ), on enregistre la
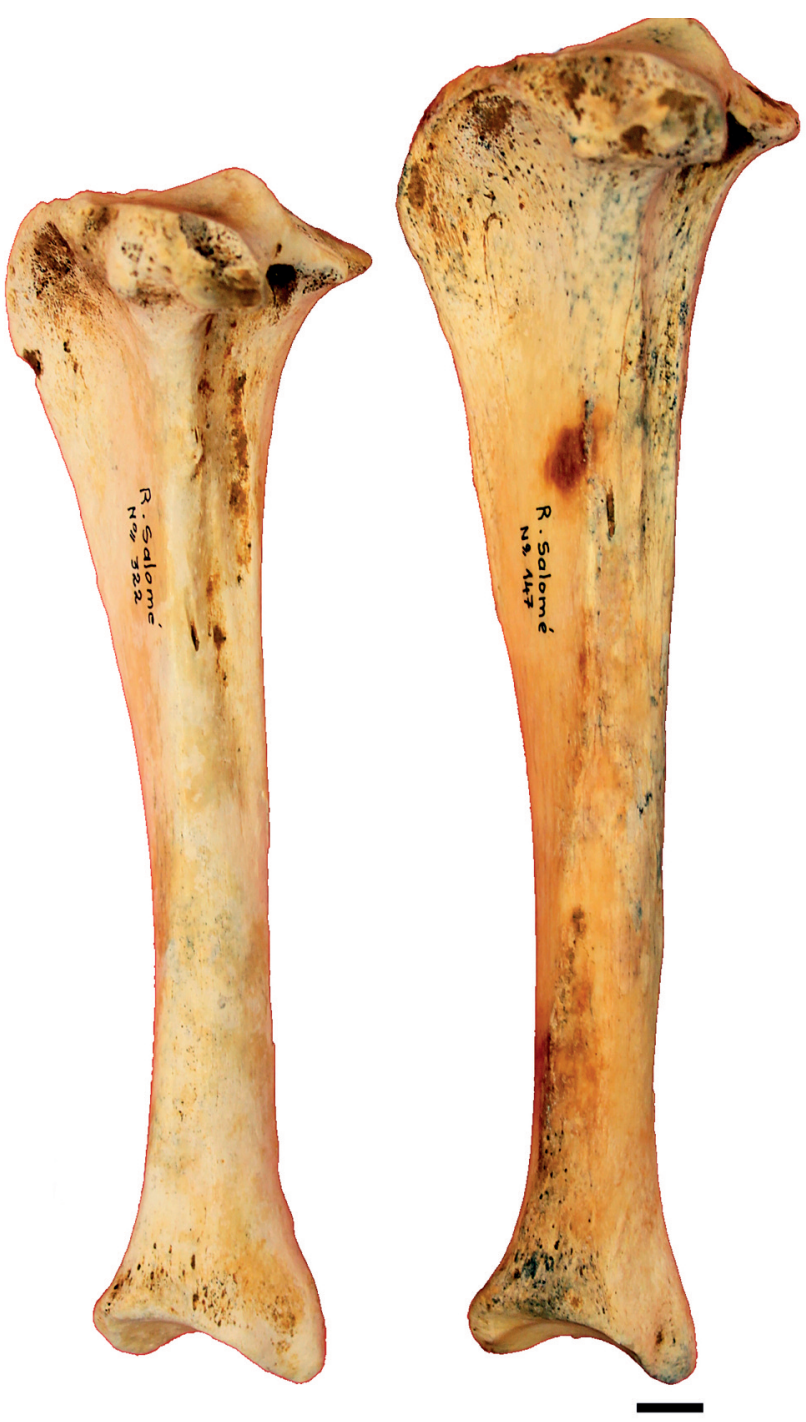

Figure 6 - Tibia d'hyène du réseau Salomé. De gauche à droite : $n^{\circ} 322, n^{\circ}$ 147. Photographies JBF.

Echelle : $10 \mathrm{~mm}$.

Figure 6 - Hyena tibia from Réseau Salomé. From left to right : $n^{\circ} 322, n^{\circ}$ 147. Pictures JBF. Bar scale : $10 \mathrm{~mm}$

plus grande diversité de traces chez l'ours avec six types reconnus contre quatre chez l'hyène. Les traces de mâchonnement (chewing) sont les plus fréquentes tant chez l'ours $(n=7)$ que chez l'hyène $(n=14)$. L'ensemble des types d'éléments osseux d'ours présente des traces (tabl. 8, 10) ; les perforations (punctures) et les enfoncements (pits) sont fréquents (4 éléments altérés pour chacun de ces types de traces). La fracturation des éléments osseux se traduit par des encoches (fracturation pits) ; ces traces ont été observées sur six pièces (trois fragments de crâne, un os coxal, et deux os longs). Une tendance différente apparaît chez l'hyène. En dehors des os longs, les autres éléments sont rarement altérés (tabl. 8, 10). Quelques traces de raclement (scores $=1$ et furrows $=2$ ) ont été mises en évidence sur des os longs préalablement mâchonnés. 

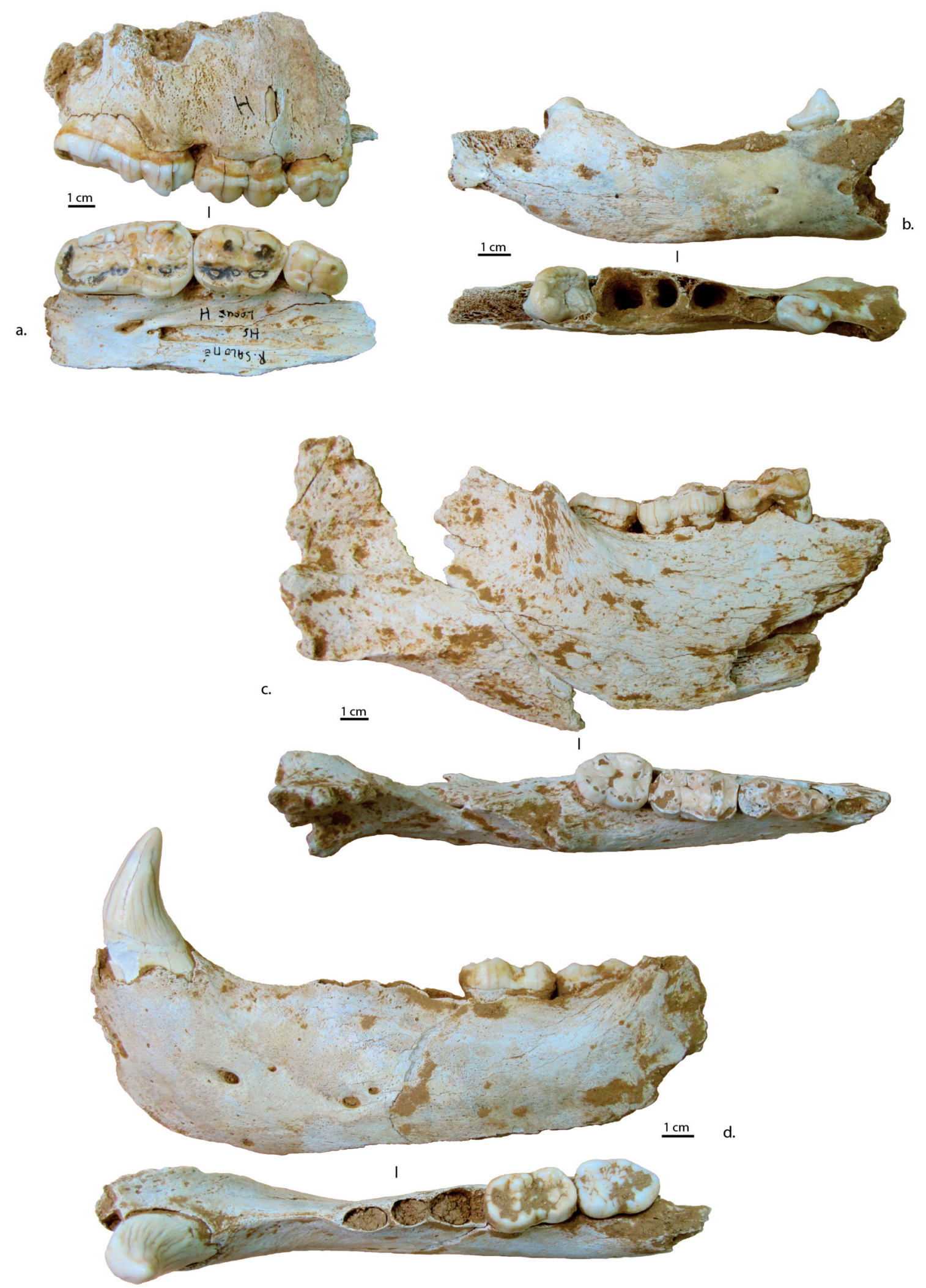

Figure 7 - Maxillaire et mandibules d'ours du réseau Salomé. De haut en bas : HS Locus H, n574A, $n^{\circ} 599, n^{\circ}$ 304. Photographies NL. Echelle : $10 \mathrm{~mm}$.

Figure 7 - Bears mandibles and maxillary from Réseau Salomé. From up to down : HS Locus H, n574A, n599, n³04. Pictures NL. Bar scale : $10 \mathrm{~mm}$. 


\begin{tabular}{|c|c|c|c|c|c|c|c|}
\hline \multirow[b]{2}{*}{$\overline{\text { Site }}$} & \multirow[b]{2}{*}{ Chrono. } & \multirow[b]{2}{*}{ Quantif. } & \multicolumn{5}{|c|}{ Matériel crânien (dentaire) } \\
\hline & & & Stade 1 & Stade 2 & Stade 3 & Stade 4 & Stade 5 \\
\hline Réseau Salomé & OIS3? & NR & 3 & 4 & 14 & 12 & 1 \\
\hline Réseau Salomé & OIS3? & $\%$ NR & 9 & 12 & 41 & 35 & 3 \\
\hline Réseau Salomé & OIS3? & NMI & 2 & 1 & 2 & 3 & 1 \\
\hline Réseau Salomé & OIS3? & $\% \mathrm{NMI}$ & 22 & 11 & 22 & 33 & 11 \\
\hline Fouvent & OIS3 & NMI & 37 & 18 & 25 & 19 & 15 \\
\hline Fouvent & OIS3 & $\% \mathrm{NMI}$ & 32 & 16 & 22 & 17 & 13 \\
\hline Conives & OIS3 & NMI & 9 & 7 & 7 & 7 & 4 \\
\hline Conives & OIS3 & $\% \mathrm{NMI}$ & 26 & 21 & 21 & 21 & 13 \\
\hline Artenac c.10 & OIS5c & NMI & 1 & 3 & 5 & 5 & 6 \\
\hline Artenac c.10 & OIS5c & $\% \mathrm{NMI}$ & 5 & 15 & 25 & 25 & 30 \\
\hline Peyre & OIS5e? & NMI & 12 & 6 & 5 & 1 & 3 \\
\hline Peyre & OIS5e? & $\% \mathrm{NMI}$ & 44 & 22 & 19 & 4 & 11 \\
\hline GAP RS & OIS5 & NMI & 5 & 4 & 6 & 3 & 3 \\
\hline GAP RS & OIS5 & $\% \mathrm{NMI}$ & 24 & 19 & 29 & 14 & 14 \\
\hline
\end{tabular}

\begin{tabular}{cccc}
\hline \multicolumn{5}{c}{ Matériel postcrânien } \\
\hline Néonatal & Juvénile & Sub-adulte & Adulte (+) \\
\hline- & 16 & 1 & 78 \\
- & 17 & 1 & 82 \\
- & 1 & 1 & 3 \\
- & 20 & 20 & 60 \\
\hline- & 6 & 3 & 5 \\
- & 43 & 21 & 36 \\
\hline- & 2 & 1 & 3 \\
- & 33 & 17 & 50 \\
\hline- & 1 & 1 & 3 \\
- & 20 & 20 & 60 \\
\hline 2 & 3 & 1 & 1 \\
29 & 43 & 14 & 14 \\
\hline 1 & 3 & 3 & 4 \\
9 & 27 & 27 & 36 \\
\hline
\end{tabular}

Tableau 6 - Structure de mortalité des hyènes d'après les restes dentaires et post-crâniens (NR,\%NR, NMI,\%NMI).

Table 6 - Hyena mortality structure according to the dental and postcranial remains (NISP, \%NISP, MNI, \%MNI).
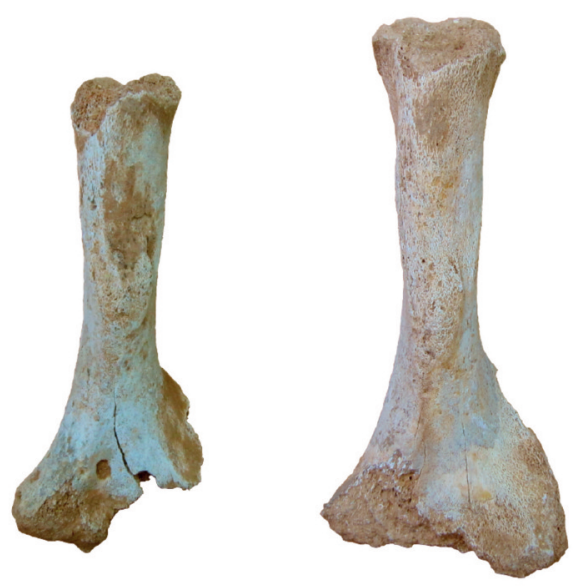

$\underline{1 \mathrm{~cm}}$
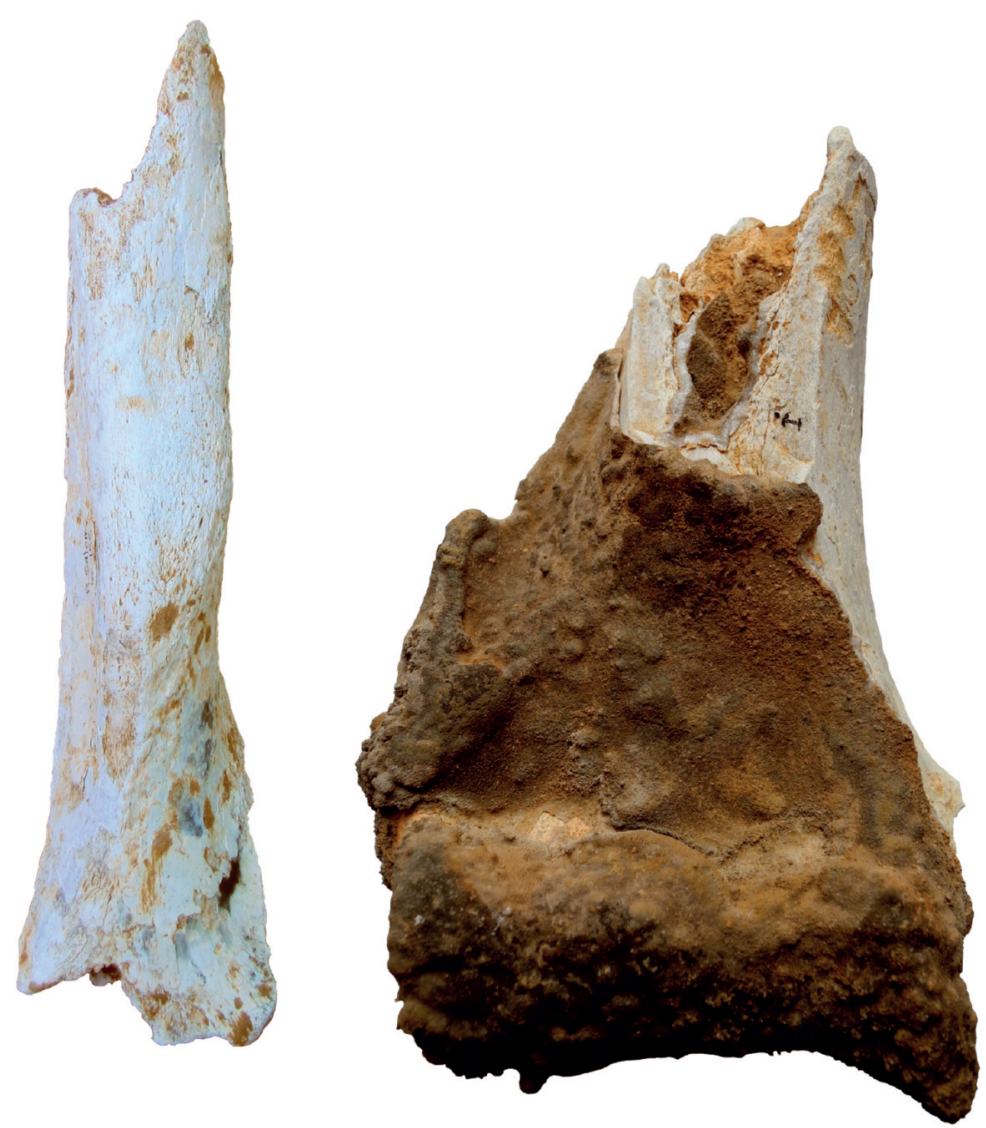

Figure 8 - Humérus d'ours du réseau Salomé. De gauche à droite : n515, n¹24, n¹55, HS Locus I. Photographies NL et MP. Échelle : $10 \mathrm{~mm}$.

Figure 8 - Bear humerus from Réseau Salomé. From left to right : $n^{\circ} 515, n^{\circ} 124, n^{\circ} 155$, HS Locus I. Pictures NL and MP. Bar scale : $10 \mathrm{~mm}$. 


\begin{tabular}{lccccc}
\hline Elément & Néonatal & Juvénile & Sub-adulte & Adulte (+) & NRT \\
\hline humérus & - & 2 & - & 2 & 4 \\
radius & - & 3 & 2 & - & 5 \\
ulna & - & 2 & - & 1 & 3 \\
fémur & - & 1 & 3 & & 4 \\
tibia & 1 & 2 & - & - & 3 \\
fibula & - & - & - & 1 & 1 \\
\hline NR os longs & $\mathbf{1}$ & $\mathbf{1 0}$ & $\mathbf{5}$ & $\mathbf{4}$ & 20 \\
\%NR os longs & $\mathbf{5 \%}$ & $\mathbf{5 0} \%$ & $\mathbf{2 5 \%}$ & $\mathbf{2 0 \%}$ & - \\
\hline métapodes & - & 2 & 11 & 10 & 23 \\
\hline \%NR métapodes & - & $\mathbf{9 \%}$ & $\mathbf{4 8 \%}$ & $\mathbf{4 3 \%}$ & - \\
\hline
\end{tabular}

Tableau 7 - Structure de mortalité des ours d'après les restes post-crâniens (NR,\%NR).

Table 7 - Bear mortality structure according to the postcranial remains (NISP, \%NISP).

\begin{tabular}{lcccc} 
& \multicolumn{4}{c}{ Ursus spelaeus } \\
\cline { 2 - 5 } Elément & NRT & NME & NR frais & NR traces \\
\hline Crâne & 31 & 9 & 2 & 5 \\
Max. & 0 & 0 & - & - \\
Dent & N/A & N/A & N/A & N/A \\
Mand. & 7 & 4 & 2 & - \\
Cerv. & 2 & 2 & - & - \\
Thor. & 4 & 4 & - & - \\
Lomb. & 3 & 3 & - & - \\
Caud. & 4 & 4 & - & - \\
Vert. & 6 & 3 & - & - \\
Côte & 3 & 2 & -- & - \\
Scap. & 4 & 2 & - & - \\
Hum. & 12 & 9 & 4 & 2 \\
Rad. & 6 & 5 & - & 1 \\
Ulna & 6 & 6 & - & - \\
Carp. & 7 & 7 & - & - \\
Mtc & 10 & 9 & - & - \\
Coxal & 5 & 5 & - & 1 \\
Fem. & 11 & 7 & 3 & 1 \\
Tib. & 7 & 6 & 2 & 2 \\
Fib. & 2 & 2 & - & 1 \\
Tars. & 6 & 6 & - & - \\
Calca. & 8 & 8 & - & 3 \\
Talus & 3 & 3 & - & - \\
Mtt & 4 & 4 & - & - \\
Phal. 1 & 14 & 14 & - & 1 \\
Phal. 2 & 17 & 17 & - & - \\
Phal. 3 & 18 & 18 & - & - \\
\hline & & & & \\
\hline
\end{tabular}

\begin{tabular}{cccc}
\hline \multicolumn{5}{c}{ Crocuta crocuta spelaea } \\
\hline NRT & NME & NR frais & NR traces \\
\hline 2 & 2 & - & - \\
3 & 3 & - & - \\
28 & 28 & - & - \\
3 & 3 & 1 & 1 \\
4 & 4 & - & - \\
4 & 4 & - & - \\
5 & 5 & - & - \\
5 & 5 & - & - \\
0 & 0 & - & - \\
1 & 1 & - & - \\
0 & 0 & - & - \\
3 & 3 & 1 & 2 \\
6 & 5 & 2 & 2 \\
0 & 0 & - & - \\
12 & 12 & - & - \\
10 & 10 & 4 & 5 \\
3 & 3 & - & 1 \\
5 & 4 & 1 & 3 \\
2 & 2 & - & - \\
1 & 1 & - & - \\
2 & 2 & - & - \\
2 & 2 & - & - \\
3 & 3 & - & - \\
11 & 11 & - & 1 \\
6 & 6 & - & - \\
2 & 2 & - & - \\
1 & 1 & - & - \\
\hline
\end{tabular}

Tableau 8 - Distribution squelettique (NR et NME associé) des restes d'ours et d'hyène.

Table 8 - Bears and hyenas skeletal part distribution (NISP and related MNE). 


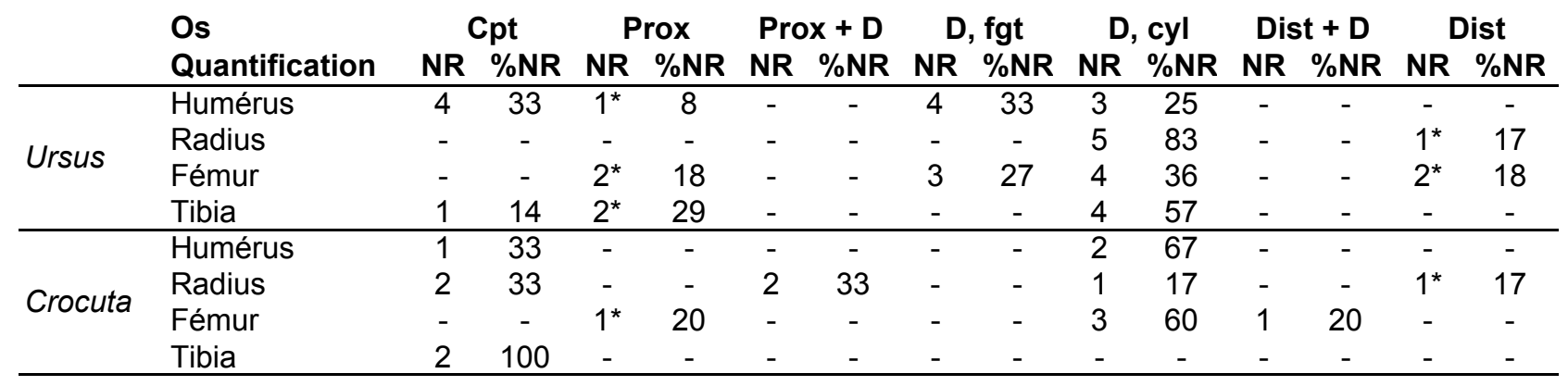

Tableau 9 - Fragmentation des os longs. NR, Nombre de restes ; \%NR, Fréquence du Nombre de restes par os long. *=épiphyse nonsoudée.

Table 9 - Long bone fragmentation. NISP and \%NISP for each limb bone. *=unfused end.

\begin{tabular}{|c|c|c|c|c|c|c|c|c|c|}
\hline Espèce & Os & Chewing & Pit F & Pit & Puncture & Score & Furrow & Crenulated & Chipping \\
\hline \multirow{4}{*}{ Ursus } & Cran/dent & - & 3 & 2 & 1 & - & - & - & - \\
\hline & Os plat & - & 1 & - & - & - & - & - & - \\
\hline & Os long & 5 & 2 & 1 & 2 & - & - & 1 & 2 \\
\hline & Os compact & 2 & - & 1 & 1 & - & - & - & - \\
\hline \multirow{3}{*}{ Crocuta } & Cran/dent & 1 & - & - & - & - & - & - & - \\
\hline & Os plat & 1 & - & - & - & - & - & - & - \\
\hline & Os long & 12 & 1 & - & - & 1 & 2 & - & - \\
\hline
\end{tabular}

Tableau 10 - Traces de consommation en fonction du type d'élément squelettique altéré.

Table 10 - Consumption marks according to each modified skeletal part.

\subsection{Analyse pollinique des coprolithes et contexte paléoenvironnemental}

Quatre coprolithes ont été sélectionnés pour l'analyse pollinique (fig. 10). Le tableau 11 donne le bilan des comptages réalisés. Le coprolithe AC-100 (B6-452) est stérile, ce qui s'explique peut-être par sa faible masse. Le plus riche (AC-102) ne contient que 24 grains de pollen. Onze taxons sont représentés sur l'ensemble des échantillons. Les arbres sont surtout des conifères : pin (Pinus) et cèdre (Cedrus), ce dernier présent seulement dans le coprolithe AC-101 (C3-21). Le chêne (Quercus) est le seul feuillu noté. Les herbacées sont faiblement représentées, sauf dans $\mathrm{AC}-102$ où les Poaceae et les Cichorioideae dominent. Ce spectre pollinique dispose d'un trop faible nombre de grains et ne permet donc pas de donner une interprétation précise, ni en termes de paysage ni en termes climatique. On peut seulement supposer un environnement présentant des zones boisées probablement dominées par des conifères, aux côtés d'espaces ouverts occupés par des herbacées.

\section{5 - Datations absolues}

Tous les échantillons ont livré un âge supérieur à 45000 ans BP (tabl. 12). Si l'on peut déplorer un manque de précision venant de ces résultats, ils ne sont pas pour autant dénués d'informations. Ils tendent à situer la formation du gisement entre le MIS 7 - qui constitue le plus ancien témoignage de la présence d'Ursus spelaeus dans le région, au sein de l'ensemble $\mathrm{F}$ de la grotte de Payre (Daujeard et Moncel 2010) - et la première moitié du MIS 3.

\section{5 - Discussion sur le statut de l'ours et la paléoécologie de l'hyène des cavernes}

Malgré l'absence d'études géomorphologiques, nous considérons a priori l'ensemble paléontologique du Réseau Salomé comme un assemblage homogène et unique. S'il semble assez clair que ces examens doivent être entrepris pour préciser le cadre contextuel de l'accumulation (e.g., mise en évidence d'une occupation unique ou multiple), il n'en reste pas moins que les études paléontologiques et taphonomiques menées, à la lumière des données paléoécologiques publiées pour d'autres gisements, nous autorisent plusieurs commentaires propres au comportement des ours et des hyènes pléistocènes.

L'échantillon osseux du réseau Salomé offre un bel exemple d'interactions entre grands prédateurs et permet de discuter de la complexité de ces relations. L'assemblage semble correspondre au schéma de site d'hivernation d'ours des cavernes au sein duquel des prédateurscharognards interviennent secondairement. Le fait que tous 

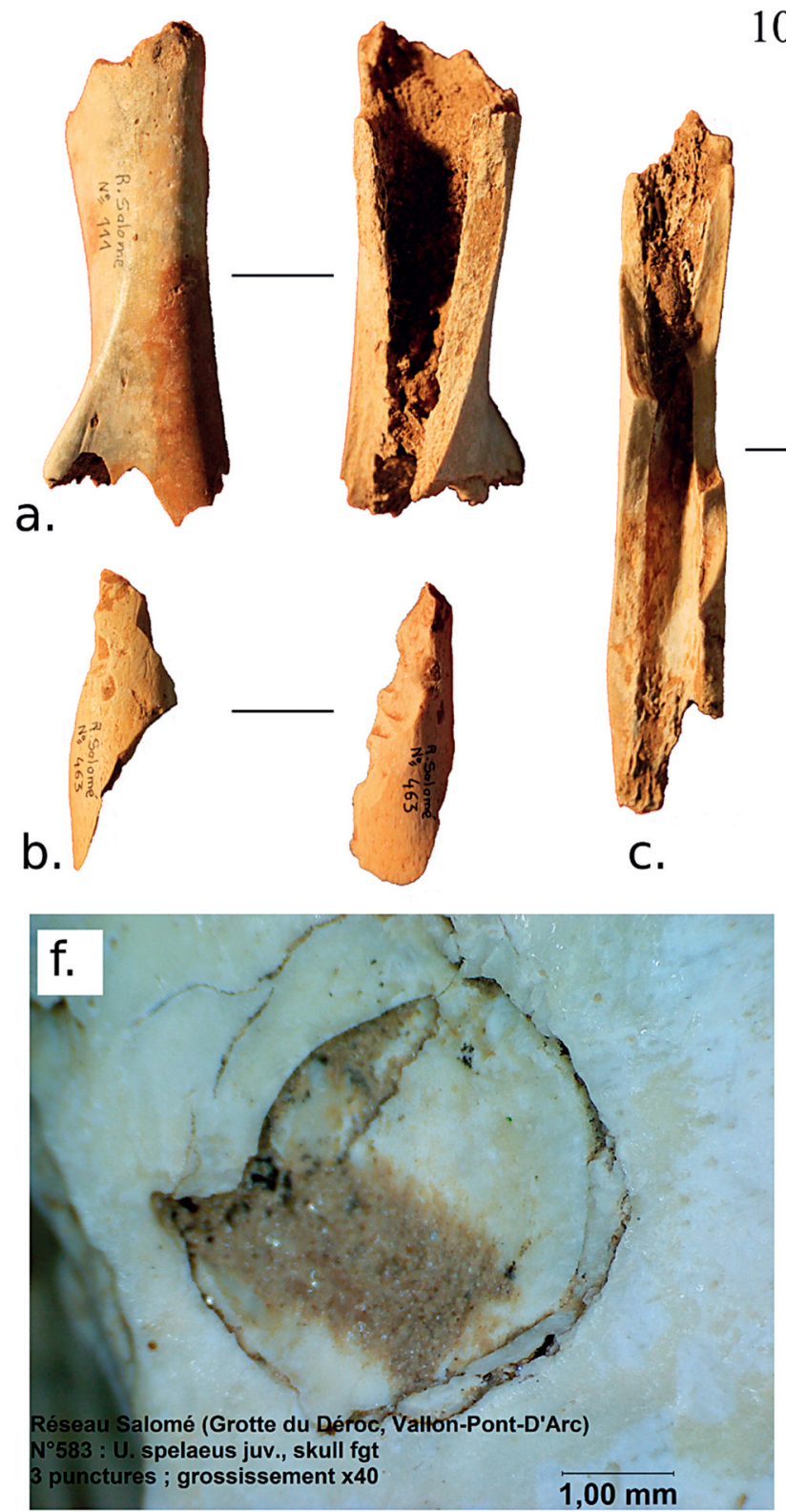

$10 \underline{\mathrm{mm}}$
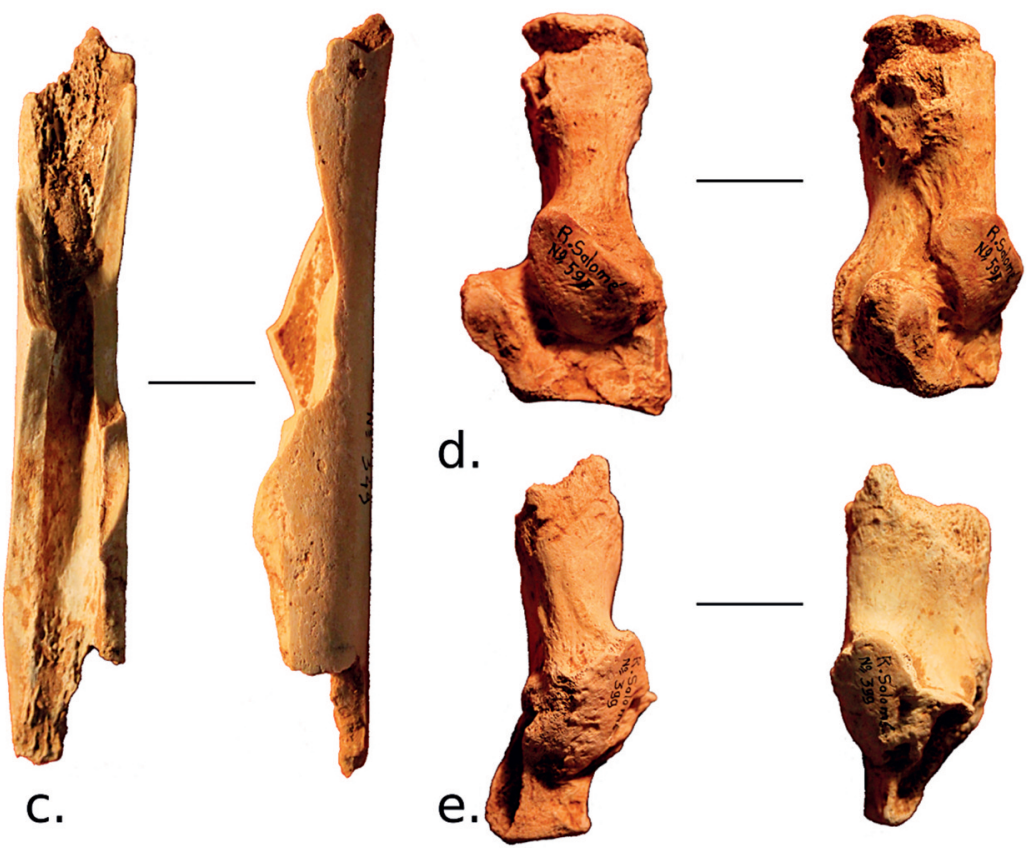

d.

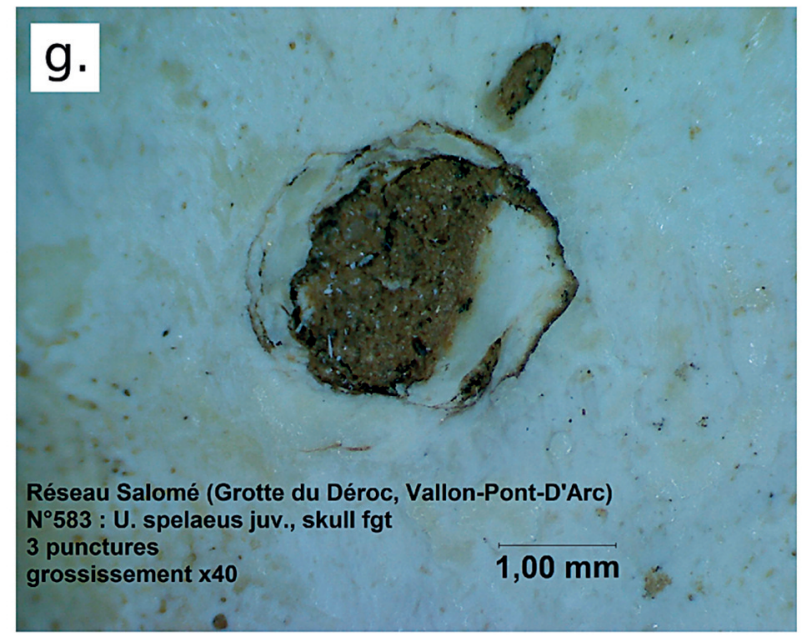

Figure 9 - Restes osseux d'ours des cavernes consommés. a. Humérus gauche de juvénile $n^{\circ} 111 ; b$. éclat diaphysaire d'os long fracturé $n^{\circ} 463 ; c$. Fémur fracturé $n^{\circ} 349 ; d$-e. Calcanéum gauche $n^{\circ} 595$ et droit $n^{\circ} 399$ rongés ; $f$-g. Détail des traces de dents. Photographies JBF.

Figure 9 - Consumed bears remains. a. Left juvenile humerus $n^{\circ} 111 ; b$. Long bone shaft fragment $n^{\circ} 463 ; c$. Fractured femur $n^{\circ} 349$; $d$-e. Left $n^{\circ} 595$ and right $n^{\circ} 399$ chewed calcanei ; f-g. Tooth mark details. Pictures JBF.

les stades ontogéniques des ours soient représentés (cf. les différents stades observés à partir des 4 humérus exploitables, fig. 8), ainsi d'ailleurs que tous les éléments squelettiques aient été recueillis, témoignent bien que le site a servi de grotte d'hivernation et que certains y sont morts pendant cette phase (suite à l'hivernation ou la prédation ou un conflit inter- ou intra-spécifique ?).
De plus, même si l'occurrence des traces peut être dépendante de la taille de l'échantillon, les différences relevées ici peuvent être le résultat d'une exploitation différentielle des carcasses en fonction de la taille de la proie et du mode d'acquisition (prédation vs. charognage).

La présence de l'hyène suggère une incursion secondaire du prédateur (en vue de la consommation des ours morts 
a.

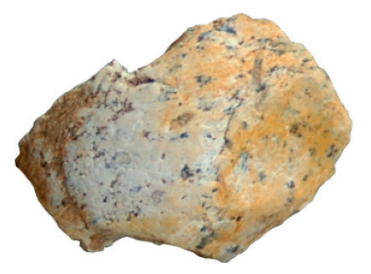

b.

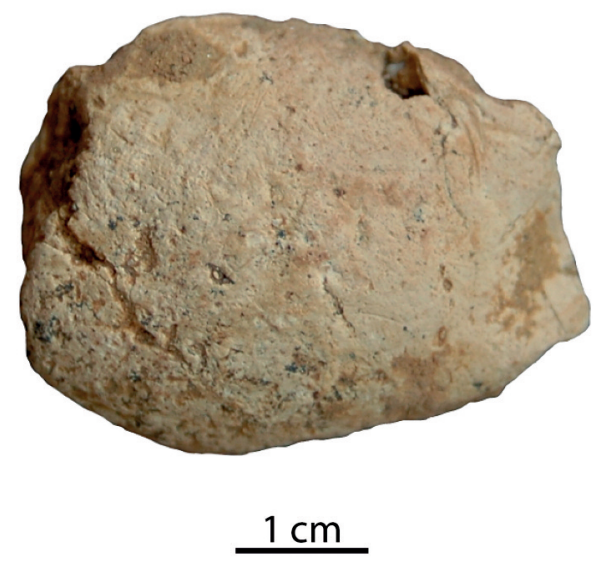

Figure 10 - Coprolithes du réseau Salomé analysés. a. $n^{\circ} 452$; b. $n^{\circ}$ 209. Photographies JA, modifiées par NL.

Figure 10 - Studied coprolites from Réseau Salomé. a. $n^{\circ} 452$; b. $n^{\circ} 209$ Pictures JA, modified by NL.

naturellement ? Ou d'une occupation du réseau profond de la cavité lors des phases hivernales ?). La structure de mortalité des hyènes se distingue de la mortalité dite naturelle observée dans les repaires d'hyènes pléistocènes (e.g., Fouvent, Peyre). La représentation importante des animaux ayant atteint a minima l'âge adulte plaide a priori en faveur de la fréquentation épisodique de la cavité. Alors que certains gisements, dont la fonction de grotte-repaire d'hyène est avérée, comportent des restes de nouveauxnés (Peyre et Le Grand Abri aux Puces réseau supérieur ; utilisation de la cavité pour la mise-bas) ces derniers sont totalement absents du réseau Salomé. Cependant, dans le registre moderne, les jeunes ne quittent pas le repaire avant l'âge de 30 mois (Kruuk 1972 ; Mills 1990) ; un épisode d'occupation de la cavité comme repaire (pour la mise-bas) par l'hyène (en raison de la présence de ces deux juvéniles de moins d'un an) ne peut donc pas être complètement exclu.

Les analyses polliniques réalisées nous éclairent un peu plus sur le comportement de l'hyène. En effet, le faible nombre de grains de pollen trouvés dans les coprolithes du réseau Salomé s'explique peut-être par une saison défavorable à la production massive de pollen, ou le jeûne de l'animal. Cette analyse vient compléter les études menées précédemment sur des coprolithes issus de gisements proches et approfondir notre connaissance de la paléoécologie de l'hyène des cavernes dans cette partie du sud-est de la France. À la grotte des Deux Ouvertures (Saint-Martin-d'Ardèche), en particulier, trois coprolithes découverts au sein d'un remplissage daté entre 37100 et 31900 cal. BP (MIS 3), ont livré des spectres polliniques révélant des conditions climatiques froides et sèches, où dominent les herbacées steppiques (Poacae et Artemisia). Les taxons arborés, peu nombreux, sont représentés par Pinus, Betula et Ephedra (Argant et Philippe 2011 ; Argant 2014). Les données polliniques du réseau Salomé suivent donc la même tendance et attestent, une fois encore, de la fréquentation des cavités profondes par les hyènes au cours des phases froides. La question d'un comportement troglophyle de l'hyène des cavernes se pose. Le prédateur semble occuper systématiquement les réseaux profonds lors de ces périodes aux conditions climatiques difficiles (MIS 3). Ce Carnivore, beaucoup plus abondamment mis au jour que d'autres taxons comme le lion, le léopard ou le loup, semble bien avoir des mœurs troglophyles nettement plus développés lors de ces phases climatiques posant la question de son éthologie (vers une hivernation de l'hyène des cavernes ?).

\begin{tabular}{lcccc}
\hline Coprolithes & AC99 & AC100 & AC101 & AC102 \\
\hline Réf. ARPA & $\mathbf{5 9 1 5}$ & $\mathbf{5 9 1 6}$ & $\mathbf{5 9 1 7}$ & $\mathbf{5 9 1 8}$ \\
\hline Conifère indét. & 1 & & & \\
Pinus & 1 & & 4 & 9 \\
Cedrux & & & 2 & \\
Quercus & & & 1 & \\
AP & $\mathbf{2}$ & $\mathbf{0}$ & $\mathbf{7}$ & $\mathbf{9}$ \\
\hline NAP & $\mathbf{3}$ & $\mathbf{0}$ & $\mathbf{2}$ & $\mathbf{1 5}$ \\
Spores & $\mathbf{0}$ & $\mathbf{0}$ & $\mathbf{1}$ & $\mathbf{0}$ \\
\hline Poaceae & 1 & & & 5 \\
Cichorioideae & 1 & & & 6 \\
Rumex & & & & 1 \\
Chenopodiacea & 1 & & 1 & 2 \\
Caryophyllaceae & & & 1 & 1 \\
Dipsacaceae & & & 10 & \\
\hline Spore mono. & & $\mathbf{0}$ & $\mathbf{1 0}$ \\
\hline Total & $\mathbf{5}$ & &
\end{tabular}

Tableau 11 - Contenu pollinique des coprolithes du réseau Salomé. $A P=$ Arboreal Pollen, NAP=Non Arboreal Pollen.

Table 11 - Pollen content of Réseau Salomé coprolites. $A P=$ Arboreal Pollen, NAP=Non Arboreal Pollen. 


\begin{tabular}{lllll}
\hline Espèce & Nature & Localisation - réf. Fouille & Datation & Référence labo \\
\hline Capra ibex & scapula & locus $\mathrm{F}$ & $>45000 \mathrm{BP}$ & Lyon-10607 (SacA35160) \\
Crocuta crocuta spelaea & fémur & carré B4 $-\mathrm{n}^{\circ} 58$ & $>45000 \mathrm{BP}$ & Lyon-10608 (SacA35161) \\
Crocuta crocuta spelaea & métapode & carré B6 $-\mathrm{n}^{\circ} 497$ & $>45000 \mathrm{BP}$ & Lyon-10609 (SacA35162) \\
Ursus spelaeus & humérus & carré B1 $-\mathrm{n}^{\circ} 10$ & $>45000 \mathrm{BP}$ & Lyon-10610 (SacA35613) \\
Ursus spelaeus & métapode & carré B6 $-\mathrm{n}^{\circ} 411$ & $>45000 \mathrm{BP}$ & Lyon-10611 (SacA35164) \\
\hline
\end{tabular}

Tableau 12 - Synthèse des datations absolues (14C AMS).

Table 12 - Radiocarbon datings (14C AMS).

La place de l'ours au sein des assemblages ostéologiques, paléontologiques et archéologiques, a été largement discutée depuis près d'un siècle. Tantôt prédateur aux mœurs cannibales (e.g., Lolliot \& Philippe 2004 ; Fourvel et al. 2012), tantôt proie des hommes (e.g., Münzel \& Conard 2004) ou des Carnivores (e.g. Fourvel et al. 2014b) voire objet de « culte » supposé (e.g., Fosse \& Philippe 2005 ; Cavanhié 2011), le statut de l'ours n'est pas si clair qu'il y paraît a priori et cet animal n'est plus le simple occupant occasionnel des cavités pour l'hivernation.

Si les représentations des grands Carnivores, et en particulier de l'ours, de l'hyène et du lion, sont relativement rares dans le registre de l'art pariétal (Sauvet 1988 ; ManEstier 2011), quelques exemples permettent néanmoins de documenter la présence de ces animaux dans l'environnement animal local au Pléistocène supérieur et de compléter les données relatives à la biodiversité, issues de contextes paléontologiques et archéozoologiques. La grotte Chauvet est sans doute le meilleur exemple en ce sens, son bestiaire ayant livré 75 représentations de lion, 16 d'ours, ainsi qu'une panthère (Clottes et Azéma 2005 ; Man-Estier 2011). Ces figures s'inscrivent dans un contexte chronologique rapporté à deux phases de fréquentations de la cavité, une aurignacienne entre 37000 et 33500 ans cal. BP, une seconde gravettienne entre 31000 et 28000 ans cal. BP (Quilès et al. 2016).

Le statut de l'ours comme part du spectre de proies des communautés humaines est bel et bien avéré. Les exemples sont nombreux (e.g., Biache-Saint-Vaast in Auguste, 1988, 1995 ; Le Regourdou in Cavanhié, 20092010). À Font-de-Gaume III (Dordogne), l'examen archéozoologique des restes d'ours atteste d'une exploitation épisodique des carcasses de ces animaux (a priori morts au cours de l'hivernation) par les groupes humains (Armand, Plassard et Prat 2003). À l'inverse, des restes d'ours ont pu être introduits par l'Homme au sein des assemblages osseux, comme c'est le cas pour les restes d'ours brun Ursus arctos exploités à l'Abri Castanet (Dordogne) (Armand 2006). En ce qui concerne le réseau Salomé, aucun indice de présence humaine n'a été mis en évidence ; aucune industrie lithique ni traces de boucherie n'ont été observées sur les vestiges osseux.

Les travaux les plus récents ont cherché à caractériser le statut de l'ours comme proie de l'hyène en contexte de grotte-repaire (Fourvel et al. 2014b). Les éléments osseux d'ours accumulés par l'hyène sont généralement peu nombreux (e.g., 13 NR aux Rochers-de-Villeneuve in Beauval \& Morin 2010, 23 à Fouvent in Fourvel et al. 2014a ; Fourvel et Lateur 2015). Le réseau Salomé, tant par la structure de population des hyènes que celle des ours, l'abondance du matériel, la distribution squelettique et la diversité des traces, ne semble pas faire écho à ce schéma et confirme bien son statut de grotte à ours avec un impact secondaire des Carnivores.

La consommation d'ours au sein des sites à ours (grotte à hivernation) semble être le résultat de deux principaux intervenants : l'ours lui-même et des Carnivores (Félidés, Canidés, Hyénidés) intervenant secondairement. Le cannibalisme est un fait reconnu chez les ours fossiles. Parmi les cas les plus typiques, les gisements du MontVentoux (MV4 et MV6 principalement) présentent d'importantes accumulations d'ours bruns, Ursus arctos, formées par des chutes récurrentes de ceux-ci dans un puits au fond des cavités (Crégut et al. 2005). Les individus qui ont survécu à la chute ont, avant leur mort, consommé les restes de leurs congénères morts avant eux (Crégut \& Fosse 2001). Le gisement de la Balme-à-Collomb (Savoie), grotte occupée par des générations d'ours des cavernes, reflète ce cannibalisme avec la présence abondante d'os impactés (Lolliot \& Philippe 2004). Ce phénomène de cannibalisme à la Balme-à-Collomb a été interprété comme un moyen d'emmagasiner de l'énergie, soit avant l'hivernation, soit au réveil des animaux. Le gisement de Coro Tracito (Espagne) est en tous points comparable à la Balme-à-Collomb (Rabal Garcés 2013). Ce site monospécifique (Ursus spelaeus) a permis de préciser la paléoécologie de l'ours des cavernes et de décrire les stigmates issus de la consommation des ours par les ours eux-mêmes. Par extension, on ne peut exclure le fait que les ours du réseau Salomé aient consommé des ours morts naturellement dans la cavité, même si ce fait reste assez difficile à mettre en évidence dans ce contexte où l'ours n'est pas l'unique prédateur.

II est important de souligner la présence courante, si ce n'est récurrente, d'autres (nombreuses et variées) espèces de Carnivores parmi lesquelles des Canidés, des Hyénidés et des Félidés. La grotte de Bourdettes (Lot-et-Garonne) en est le parfait exemple (Discamps et al. 2012). Ce gisement semble relativement comparable au réseau Salomé avec près de $95 \%$ des restes attribué à l'ours (NR=7520, $\mathrm{NMl}=126)$, la présence non-négligeable d'hyène $(\mathrm{NR}=130)$ 
mais aussi du lion $(\mathrm{NR}=1)$ et du loup $(\mathrm{NR}=6)$. L'étude taphonomique menée sur ce site témoigne d'une consommation secondaire des Ursidés par d'autres prédateurs (l'hyène apparait selon les auteurs comme l'intervenant principal). Nous regretterons cependant que les restes d'ours n'aient pas été intégrés à l'étude taphonomique. Le rôle de l'hyène sur la consommation / le charognage des carcasses d'ours lors de l'hivernation a été décrit dans plusieurs sites allemands (Sophie's Cave in Diedrich 2013 ; Teufelskammer cave ou Perick cave in Diedrich 2014) et tchèques (Sloup cave in Diedrich 2014). Cependant, l'absence de données quantifiées n'autorise aucune réelle comparaison avec le réseau Salomé.

La grotte d'Azé en Bourgogne est un exemple caractéristique de l'incursion de grands Carnivores (lion) au sein de la cavité pour consommer des ours (Argant 1991, 2000). Les ensembles I et II d'Artenac (Charente) constituent des exemples similaires à Azé, où des jaguars européens Panthera gombaszoegensis ont chassé ou charogné Ursus deningeri (Tournepiche 1996 ; Fourvel et al. 2012, 2014b). En ce sens, la présence de la phalange de lion au sein du réseau Salomé, bien que marginale, n'en reste pas moins intéressante, en particulier pour ce qui est des relations paléoécologiques (prédateurs-proies ?) entre les grands félins pléistocènes et les Ursidés (synthèse in Fourvel et al. 2014b). Cette simple pièce atteste de la présence du Félidé et, par conséquent, sans autre donnée de terrain ou ostéologique, son rôle (même minime) dans la mise en place et/ou dans la modification du dépôt osseux ne peut être exclu. Par ailleurs, le même commentaire s'applique au renard. En effet, même si ce dernier n'a pas été retrouvé en stratigraphie, sa présence reste néanmoins un témoin de son passage et de son potentiel impact taphonomique sur l'assemblage initial.

\section{6 - Conclusion générale}

Le gisement paléontologique du réseau Salomé apparaît comme un site d'importance pour la connaissance des faunes de Carnivores pléistocènes. Si en effet les grottes à ours ne sont pas rares, l'association (quasi) stricte de l'ours et de l'hyène est nettement moins fréquente. En ce sens, ce gisement est un parfait exemple des interactions entre les communautés de grands Carnivores pour l'accès aux ressources alimentaires et à l'habitat (éléments conditionnant par là même les conflits entre communautés humaines et Carnivores au cours du Paléolithique).

En dépit de datations peu précises et de l'absence d'analyses sédimentologiques, il semble a priori que la fréquentation du réseau Salomé par les différents Carnivores identifiés sur le site soit contemporaine (même si des analyses complémentaires, dont examen géomorphologique-sédimentologique, devront préciser ce point). La structure de la population d'ours et l'analyse taphonomique suggèrent que le réseau Salomé a essentiellement servi de gîte d'hivernation pour les ours des cavernes, dans lequel des Carnivores sont venus tirer profit de carcasses d'ours morts naturellement et, ce faisant, ont modifié une partie de l'assemblage osseux initial. Parmi ces Carnivores, l'hyène semble être le principal agent responsable de ces modifications, au regard de la fragmentation du matériel et des morpho-types osseux. Mais nous ne pouvons pas écarter les possibilités qu'un cannibalisme de l'ours des cavernes et/ou un impact par le lion des cavernes aient aussi contribué à modifier l'assemblage. Quoi qu'il en soit, à ce jour, aucun élément de chronologie ne nous permet d'identifier plusieurs phases de fréquentations par les Carnivores très espacées dans le temps.

En matière d'étude paléontologique, le réseau Salomé présente une réelle richesse pour la connaissance de l'hyène des cavernes. L'abondance et la qualité de la préservation des éléments post-crâniens nous permettent de disposer d'un important échantillon autorisant d'une part la description morphométrique de ces vestiges trop rarement étudiés et, d'autre part, d'intégrer ces éléments dans une réflexion plus large sur l'évolution des Hyénidés pléistocènes. En définitive, l'accumulation du réseau Salomé, tout à fait originale par sa composition et son état de conservation, est riche d'informations paléontologiques et paléoécologiques. Ce gisement nous aura permis ainsi de discuter du statut paléoécologique des différents grands prédateurs (ours et hyène) et d'apporter des informations inédites sur les relations inter- et intra-spécifiques, ces conflits étant autant d'informations ouvrant sur les phénomènes d'interactions et de compétitions entre les Carnivores fossiles et les groupes humains paléolithiques qui partagent le même fonds écologique.

\section{Remerciements}

Nous tenons à remercier sincèrement les membres du spéléo-club d'Aubenas qui sont à l'origine de la découverte et qui nous ont épaulés tout au long de la fouille, assurant l'organisation logistique spéléologique de l'opération et qui ont suivi avec intérêt nos travaux. La bonne conservation du site est le fait de leur vigilance et de leur sérieuse expérience! Nous remercions André Massot, propriétaire du terrain, qui nous a autorisés à réaliser les fouilles. Nos remerciements s'adressent aussi à Bernard Gély, du Service régional de l'archéologie Auvergne-Rhône-Alpes qui, après avoir été averti de la découverte, nous a sollicités pour ces recherches. Nous remercions bien sûr toutes les personnes qui ont participé à la fouille : Antonin et Daniel Ariagno, Julien Monney, Jacques Romestan et Sonia Stocchetti. Enfin, nous tenons à adresser nos plus sincères remerciements à l'ensemble de l'équipe de la Cité de la Préhistoire d'Orgnac-l'Aven sans laquelle ce travail n'aurait pu voir le jour. Nous remercions Patricia Guillermin, conservatrice de la Cité de la Préhistoire, et Robin Furestier, responsable des collections et de la recherche, pour nous avoir grandement facilité l'accès aux collections. Enfin, nous adressons notre gratitude à $\mathrm{M}$. Jean-François Tournepiche et $M$. Jean-Baptiste Mallye pour leurs commentaires des plus pertinents et constructifs. 


\section{Références bibliographiques}

ARGANT A. 1991 - Carnivores quaternaires de Bourgogne. Lyon, Département des Sciences de la Terre. Université Claude-Bernard-Lyon 1.

ARGANT A. 2000 - Les sites paléontologiques du Pléistocène moyen en Mâconnais. Bulletin de la Société préhistorique française, 97(4), p. 609-623.

ARGANT J. 2014 - La copropalynologie : coprolithes et paléoenvironnement. Bulletin du Musée d'Anthropologie préhistorique de Monaco, 54, p. 41-46.

ARGANT J., PHILIPPE M. 2011 - L'analyse pollinique des coprolithes : un outil pour la reconstruction du paléoenvironnement. In: A. Argant, J. Argant (Eds.), Proceedings of the 16th International Cave Bear and Lion Symposium, Azé (Saône-et-Loire), 22-26 septembre 2010, Quaternaire, Hors-série (4), p. 307-318.

ARMAND D. 2006 - Abri Castanet (Dordogne, France): An Aurignacian Site with Bear Procurement. Bear Exploitation in Paleolithic Time. Scientific Annals, School of Geology Aristotle University of Thessaloniki (AUTH), Special volume 98, p. 263-268.

ARMAND D., PLASSARD F., PRAT F. 2003 - L'ours des cavernes de Font-de-Gaume III. Paleo, 15, p. 241-244.

AOURAGHE H. 1992 - Les faunes de grands mammifères du site pléistocène moyen d'Orgnac 3 (Ardèche, France). Étude paléontologique et palethnographique. Implications paléoécologiques et biostratigraphiques. Muséum national d'Histoire Naturelle, Thèse de doctorat : 492.

AUGUSTE P. 1988 - Apports paléontologiques et archéozoologiques de l'étude de la faune des grands mammifères de Biache-Saint-Vaast (Pas-de-Calais). Revue archéologique de Picardie, 1-2, p.63-68.

AUGUSTE P. 1995 - Chasse et charognage au Paléolithique moyen : l'apport du gisement de BiacheSaint-Vaast (Pas-de-Calais). Bulletin de la S.P.F., 92 (2), p. $155-167$.

BALLESIO R., BARTH P., GELY B., PHILIPPE M., ROSA M. 2003 - Contribution à l'étude des Ursidés pléistocènes des gorges de l'Ardèche et de leurs plateaux : les restes d'Ursus deningeri de la grotte des Fées, à Vallon-Pontd'Arc (Ardèche). Cahiers scientifiques du Muséum d'Histoire naturelle de Lyon (2), p. 5-51.

BALME F. 1984 - Les carnivores du gisement pléistocène supérieur de l'abri des Pêcheurs (Ardèche).Université Claude Bernard-Lyon I, Mémoire de DEA : 29.

BARYSHNIKOV G. 1999 - Chronological and geographical variability of Crocuta spelaea (Carnivora, Hyaenidae) from the Pleistocene of Russia. Deinsea, 6, p. 155-174.

BEAUVAL C., MORIN E. 2010 - Les repaires d'hyènes du Lussacois (Lussac-les-Châteaux, Vienne, France). Apport des sites des Plumettes et des Rochers-de-Villeneuve. In : J. Buisson-Catil, J. Primault (eds) Préhistoire entre Vienne et Charente. Hommes et sociétés du Paléolithique. Mémoire XXXVIII, p. 175-189.

BINFORD L. R. 1981 - Bones : Ancient men and modern myths. New York, Academic Press.

BONIFAY M.-F. 1971 - Carnivores Quaternaires du SudEst de la France. Mémoire du Muséum National d'Histoire naturelle, $377 \mathrm{p}$.

BRAIN C. K. 1981 - The hunters or the hunted? An introduction to African cave taphonomy. Chicago.

CARDOSO J. L. 1993 - La Hyène des «Oubliettes» de Gargas, Crocuta crocuta spelaea (Mammalia, Carnivora). Bulletin du Muséum National d'Histoire Naturelle $4^{e}$ série, 15, section $\mathrm{C}(1-4)$, p. 79-104.

CAVANHIÉ N. 2009-2010 - L'ours qui a vu l'homme ? Étude archéozoologique et taphonomique du site paléolithique moyen de Regourdou (Montignac, Dordogne, France). Paleo, 21, p. 39-64.

CAVANHIÉ N. 2011 - L'ours : un animal emblématique de la Préhistoire... ou du préhistorien? In : J.-P. Brugal, A. Gardeisen, A. Zucker (eds) : Prédateur dans tous leurs états. Evolution, Biodiversité, Interactions, Mythes, Symboles. XXXle rencontres internationales d'archéologie et d'histoire d'Antibes. Antibes, APDCA, p. 285-297.

CHAUVIRÉ C. 1962 - Les gisements fossilifères quaternaires de Châtillon-Saint-Jean (Drôme), Université de Lyon: Thèse de 3ème Cycle: 216.

CLOT A. 1980 - La grotte de la Carrière (Gerde, HautePyrénés, France). Stratigraphie et Paléontologie des Carnivores. Université Paul Sabatier: 239.

CLOTTES J., AZEMA M. 2005, Les félins de la grotte Chauvet. Ed. Le Seuil, 125 p.

CRÉGUT-BONNOURE E., ARGANT A., ARGANT J., AYACHE-DONAT B., BILLIOU D., BOCHERENS H., BOUCHER C., BUISSON-CATIL J., DEBARD E., FOSSE P., HANNI C., LAUDET F., MOURER-CHAUVIRÉ C., MEIN P., OBERLIN C., ORLANDO L., PHILIPPE M., QUILĖS J., THINON M. 2005 - Les cavités karstiques du Mont Ventoux (Vaucluse, France) : des ours brun (Ursus arctos L.) et des milieux d'altitude à l'Holocène. Proceedings of the 10th International Cave Bear Symposium, Mas d'Azil (Ariège, France), p. 5-18.

CRÉGUT-BONNOURE E., P. FOSSE 2001 - Holocene Brown Bears (Ursus arctos L.) in natural traps : exceptional sites of Mont Ventoux (Vaucluse, France). Cadernos 26, p. $325-340$.

DAUJEARD C., MONCEL M.-H. 2010 - On Neanderthal subsistence and land use : a regional focus on the Rhone Valley area in Southeastern France. Journal of Anthropological Archaeology 29, p. 368-391. 
DEBARD E. 1988 - Le Quaternaire du Bas-Vivarais d'après l'étude des remplissages d'avens, de porches de grottes et d'abris sous roche. Dynamique sédimentaire, paléoclimatologie et chronologie. Lyon, Documents du laboratoire de géologie de Lyon 103.

DEBARD E., PHILIPPE M. 2008 - Paléoenvironnements et faune préhistorique.In : M. Raimbault, C. Rouchouse et H. Ozil, De la Dent de Rez aux Gorges de l'Ardèche, Syndicat de Gestion des Gorges de l'Ardèche, Editions de l'lbie, Edition du Chassel, p. 113-125.

DEBARD E., FAURE M., GUERIN C., ARGANT J., JEANNET M. 1999 - Le gisement pléistocène de l'aven Flahaut à Saint-Remèze (Ardèche). Ardèche Archéologie 16, p. 1-6.

DIEDRICH C. G. 2013 - Extinctions of Late Ice Age Cave Bears as a Result of Climate/Habitat Change and Large Carnivore Lion/Hyena/Wolf Predation Stress in Europe. ISRN Zoology, Volume 2013, Article ID 138319, 25 pages, http://dx.doi.org/10.1155/2013/138319

DIEDRICH C. G. 2014 - Palaeopopulations of Late Pleistocene Top Predators in Europe: Ice Age Spotted Hyenas and Steppe Lions in Battles and Competition about Prey. Paleontology Journal, Volume 2014, Article ID 106203, 34 pages, http://dx.doi.org/10.1155/2014/106203

DISCAMPS E., BOUDADI-MALIGNE M., CHAGNEAU J., ARMAND D., GUADELLI J.-L., LENOIR M. 2012 - Ours, hommes, hyènes :qui a occupé la grotte de Bourdette (Sainte-Colombe-en-Bruilhois, Lot-et-Garonne, France). Paleo, 23, p. 117-136.

FOSSE P. 1994 -Taphonomie Paléolithique : Les grands mammifères de Soleilhac (Haute-Loire) et de Lunel-Viel 1 (Hérault). Laboratoire d'Anthropologie et de Préhistoire des Pays de la Méditerranée Occidentale, Laboratoire de Géologie du Quaternaire. Aix-Marseille, Université de Provence - Aix-Marseille I: 318.

FOSSE P., JAUZION G., MAKSUD F., QUETTIER D., QUETTIER R., ROUCH P., BESSON J.-P. 2001 - Ursidés pléistocènes des Pyrénées : éléments de paléontologie et de paléobiologie. Bulletin de la Société Préhistorique Ariège-Pyrénées LVI, p. 103-138.

FOSSE P., PHILIPPE M. 2005 - La faune de la grotte Chauvet : paléobiologie et anthropozoologie. Bulletin de la Société Préhistorique Française Travaux 6, p. 89-102.

FOURVEL J.-B. 2012 - Hyénidés modernes et fossiles d'Europe et d'Afrique : taphonomie comparée de leurs assemblages osseux. Université de Toulouse. Thèse de Doctorat: 615 , inédit.

FOURVEL J.-B., LATEUR N., PHILIPPE M. 2016 L'Hyène des cavernes, un carnivore emblématique du département de l'Ardèche et des gorges de l'Ardèche. Ardèche Archéologie 33, p. 3-10.
FOURVEL J.-B., LATEUR N. 2015 - Note sur deux mandibules de Pachycrocuta brevirostris (Aymard, 1846) du Pléistocène ancien du sud-est de la France : la grotte de la Grosse Marguerite (Aiguèze) et l'aven des Trois Pigeons (Nîmes). Paléo 26, p. 187-200.

FOURVEL J.-B., FOSSE P., FERNANDEZ P., ANTOINE P.-O. 2015 - Large mammals of Fouvent-Saint-Andoche (Haute-Saône, France): a glimpse into a Late Pleitocene hyena den. Geodiversitas 37 (2), p. 237-266

FOURVEL J.-B., FOSSE P., FERNANDEZ P., ANTOINE P.-O. 2014a - La grotte de Fouvent, dit l'Abri Cuvier (Fouvent-le-Bas, Haute-Saône, France) : analyse taphonomique d'un repaire du Pléistocène supérieur (OIS3). Paleo 25 : 79-99

FOURVEL J.-B., FOSSE P., BRUGAL J.-P., CRÉGUT E., SLIMAK L., TOURNEPICHE J.-F. 2014b Characterization of bear remains consumption by Pleistocene large carnivores (Felidae, Hyaenidae, Canidae). Quaternary International 339-340, p. 232-244.

FOURVEL J.-B., FOSSE P., BRUGAL J.-P., TOURNEPICHE J.-F., CRÉGUT E. 2012 - Consumption of Ungulate Long Bones by Pleistocene Hyaenas: a Comparative Study. Journal of Taphonomy 10(3-4), p. 249-276.

GAMBERI L., ARGANT A., ARGANT J., BARTH P., BOUDADI-MALIGNE M., BOULBES N., BRUGAL J.-P., CARAMELLI D., CONDEMI S., CREGUT-BONNOURE E., DEBARD E., ERRERA B., FARRE B., FAURE M., FERNANDEZ P., GEIGLE. M., GUERIN C., HARTERLAILHEUGUE S., JEANNET M., LATEUR N., MALLYE, J.B., MARTIN S., MONNEY J., ROMAN C., ROUSSELIERES F., SABAUT M., VALLI A. 2011 - L'aven de l'Arquet - Barjac (30). Etude d'un aven piège. ArdècheArchéologie 28, p. 3-10.

KLEIN R. G., SCOTT K. 1989 - Glacial/Interglacial Size Variation in Fossil Spotted Hyenas (Crocuta crocuta) from Britain. Quaternary Research 32, p. 88-95.

KRUUK H. 1972 -The Spotted Hyena : A Study of Predation and Social Behavior.

KURTÉN B. 1956 - The Status and Affinities of Hyaena sinensis Owen and Hyaena ultima Matsumoto. American Museum Novitates 1764, p. 1-48.

KURTÉN B. 1958 - The bears and hyaenas of the interglacials. Quaternaria 4, p. 69-81.

KURTÉN B. 1962 - The spotted hyena (Crocuta crocuta) from the middle Pleistocene of Mosbach at Wiesbaden, Germany. Commentationes Biologicae XXIV(3), p. 3-9.

KURTÉN B. 1963 - The Cave Hyena, an essay in statistical analysis. In : D. Morothwell, E. Higgs (eds) Science in Archaeology, p. 224-234. 
KURTÉN B. 1972 - Fossil Hyaenidae from the Excavations at Strànska Skàla. Strànska Skàla 1 1910-1945, Anthropos, p. 1-20.

KURTÉN B., POULIANOS A. N. 1977 - New stratigraphic and faunal material from Petralona cave, with special reference to the Carnivora. Anthropos 4, p. 47-130.

LATEUR N. 2010 - Les grands carnivores préhistoriques dans la région de Grospierres. Essai d'inventaire des gisements et premiers éléments de réflexion. Les nouveaux cahiers du Grospierrois 7, p. 7-13.

LOLLIOT S., PHILIPPE M. 2004 - Note préliminaire sur les traces de charognage affectant les ossements d'ours des cavernes, Ursus spelaeus, de la Balme à Collomb (Entremont-Le-Vieux, Savoie) ; en exemple : le cas des fémurs. Actes du 9e Symposium international sur l'ours des cavernes. Cahiers Scientifiques. Hors Série n² 2, p. 115-120.

MAN-ESTIER E. 2011 - Les Ursidés au naturel et au figuré pendant la Préhistoire. ERAUL 127, 120 p.

MICHEL V., SHEN G., SHEN C.-C., WU C.-C., VERATI C., GALLET S., MONCEL M.-H., COMBIER J., KHATIB S., MANETTI M. 2013 - Application of U/Th and 40Ar/39Ar Dating to Orgnac 3, a Late Acheulean and Early Middle Palaeolithic Site in Ardèche, France. PlosOne 8 (12), e82394. doi:10.1371/journal.pone.0082394

MILLS M. G. L. 1990 -Kalahari hyaenas: the comparative behivioural ecology of two species. London.

MONCEL M.-H., PUAUD S., DAUJEARD C., LARTIGOTCAMPIN A.-S., MILLET J.-J. THEODOROPOULOU A., CREGUT-BONNOURE E., GELY B., VERCOUTERE C., DESCLAUX E., ROGER T. et BOURGES F. 2012 - La grotte du Figuier (Saint-Martin-d'Ardèche) : bilan des travaux récents sur un site du Paléolithique moyen et supérieur de la moyenne vallée du Rhône (Sud-Est de la France). Bulletin de la Société Préhistorique française 109(1), p. 35-67.

MÜNZEL S. C., CONARD N. J. 2004 - Cave bear hunting in Höhle Fels Cave in the Ach Valley of Swabian Jura. Revue de Paléobiologie 23(2), p. 877-885.

PHILIPPE M. 2005 - La grotte Chauvet et les autres « grottes à ours » de ses environs immédiats : vallée de l'lbie, amont des gorges de l'Ardèche et Cirque d'Estre. Ardèche Archéologie 22, p. 8-19.
PHILIPPE M., FOSSE P. 2003 - La faune de la grotte Chauvet (Vallon-Pont-d'Arc, Ardèche) : présentation préliminaire paléontologique et taphonomique. Paleo 15, p. $123-140$.

PHILIPPE M., LATEUR N. 2013 - L'environnement animal des hommes préhistoriques : un regain d'intérêt et de nouvelles approches. Ardèche Archéologie 30, p. 23-27.

QUILES J. 2003 - Les Ursidae du Pléistocène moyen et supérieur en Midi méditerranéen : apports paléontologiques, biochronologiques et archéozoologiques. Muséum National d'Histoire naturelle, Thèse de doctorat : 1332.

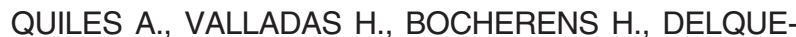
KOLIC E., KALTNECKER E., VAN DER PLICHT J., DELANNOY J.-J., FERUGLIO V., FRITZ C., MONNEY J., PHILIPPE M., TOSELLO G., CLOTTES J., GENESTE J.M. 2016 - A high-precision chronological model for the decorated Upper Paleolithic cave of Chauvet-Pont d'Arc, Ardèche, France. Proceedings of the National Academy of Sciences 113 (17), p. 4670-4675

RABAL GARCÉS R. 2013 - Estudio paleontologico de Ursus spelaeus Rosenmüller, 1794 del Pleistoceno Superior de Coro Tracito (Tella, Huesca, Espana).Universidad de Zaragoza. Tesis Doctoral: 533, unpublished.

SAUVET G. 1988 - La communication graphique paléolithique (de l'analyse quantitative d'un corpus de données à son interpretation sémiologique. L'Anthropologie 21 (1), p. 3-16.

SUTCLIFFE A. J. 1970 - Spotted Hyaena: Crusher, Gnawer, Digester and Collector of Bones. Nature 227, p. $1110-1113$.

TOURNEPICHE J.-F. 1996 - Les grands mammifères pléistocènes de Poitou-Charente. Paleo 8, p. 109-141.

TURNER A., ANTON M., WERDELIN L. 2008 - Taxonomy and evolutionary patterns in the fossil Hyaenidae of Europe. Geobios 41, p. 677-687.

VILLA P., MAHIEU E. 1991 - Breakage patterns of human long bones. Journal of Human Evolution 21, p. 27-48.

WERDELIN L., SOLOUNIAS N. 1991 -The Hyaenidae : taxonomy, systematics and evolution. Oslo. 
